\title{
Error Performance Analysis of Diffusive Molecular Communication Systems with On-Off Keying Modulation
}

\author{
Lu Shi, Student Member, IEEE and Lie-Liang Yang, Fellow, IEEE
}

\begin{abstract}
In this paper, we analyze the bit error rate (BER) of the diffusive molecular communication (DMC) systems employing on-off keying (OOK) modulation. We also analyze the BER of the OOK-modulated DMC systems with inter-symbol interference cancellation (ISIC). Our main motivation is to introduce alternative tools for analyzing and efficiently computing the BER of the DMC systems without or with ISIC. Specifically, for the OOK-modulated DMC systems without ISIC, we first derive an exact BER expression based on the Poisson modeling of DMC systems. Then, the Gaussian- and Gamma-approximation approaches are introduced to approximate the discrete Poisson distribution, and based on the approximation approaches, the corresponding BER expressions are derived. Furthermore, in order to reduce the computation complexity imposed by long ISI, we propose the Monte-Carlo, simplified Poisson, simplified Gaussian and the simplified Gamma approaches for BER computation. In the context of the OOK-modulated DMC systems with ISIC, we consider both the Poisson and Gaussian-approximation approaches for BER analysis. Again, exact and approximate BER expressions are derived under the Poisson, Gaussianapproximation, simplified Poisson and simplified Gaussian approaches. Finally, the considered approaches are compared and validated by a range of performance results obtained from evaluation of the derived expressions or by simulations. Our studies show that the alternative approaches are in general effective for providing near-accurate BER estimation.
\end{abstract}

Index Terms-Molecular communications, diffusion, concentration shift keying, on-off keying, inter-symbol interference cancellation, performance analysis, Gaussian-approximation, Gamma-approximation.

\section{INTRODUCTION}

In molecular communications (MC), information is conveyed between nano-machines with the aid of molecules [2, 3]. Recently, MC has been drawing an increasing attention in terms of its research and implementation, as witnessed, e.g., by the references $[2,4-8]$ and the references therein. In $\mathrm{MC}$, the diffusion-based molecular communications (DMC) has been recognized as one of the most practical information transmission methods, which relies on the law of diffusion for molecule propagation from (nano-)transmitters to (nano)receivers [2, 6, 9].

L. Shi and L.-L. Yang are with School of Electronics and Computer Science, University of Southampton, SO17 1BJ, UK. (E-mail: 1s25g12, 1ly@ecs.soton.ac.uk, http://www-mobile.ecs.soton.ac.uk/lly).

The work was supported in part by the EPSRC of the UK and the Innovate UK. A small part (mainly Section II-B) of the paper was published as [1] L. Shi and L.-L. Yang, "Diffusion-Based Molecular Communications: InterSymbol Interference Cancellation and System Performance," 2016 IEEE/CIC International Conference on Communications in China (ICCC), 27-29 July 2016, Chengdu, China.
As in any existing communication systems, information in DMC is delivered via the data modulation operated at transmitters and the signal demodulation (detection) carried out at receivers, and both of which are critical for achieving high-efficiency and high-reliability. Hence in literature, there are many references dedicated to the design of DMC data modulation, signal detection and transceiver optimization schemes, based on the proposed channel models and noise statistics in DMC. To be more specific, for data modulation, there are typically three basic types. The first type relies on the molecule concentration, referred to as the concentration shift keying (CSK) [10-18], which divides the molecule concentration into some levels for information configuration. The second type belongs to the pulse position modulation (PPM) [19], which delivers information by the positions of the pulses of molecule concentration. In the above two types, one type of molecules is usually used. By contrast, the third type uses multiple types of molecules, forming the so-called molecule shift keying (MoSK) $[3,14,15,20]$. Based on these basic modulation schemes, hybrid and improved modulation schemes [21-23] may be designed for different purposes, e.g., for achieving higher data rate, interference control, etc. In DMC, a typical phenomenon severely affecting communication is the intersymbol interference (ISI) generated by the long delay spread of molecular diffusion in liquid medium $[8,24]$. Hence in literature, a range of advanced detection algorithms have been proposed to mitigate the effect of ISI [1, 25-29].

Regardless of which data modulation scheme and detection scheme are employed, it is always important to predict the achievable performance of DMC systems. Hence, in this paper, we motivate to provide some approaches for analysis and evaluation of the error performance of DMC systems. More importantly, we motivate the approaches that are feasible for computation and are capable of providing accurate or nearaccurate estimation of the error performance of the DMC systems experiencing strong ISI.

In literature, there are a number of references dedicated specifically to the error rate analysis of the DMC systems with different modulation and detection schemes. Specifically, in [21], the error performance of the DMC systems with respectively three modulation schemes, namely, CSK, MoSK and the proposed so-called molecule concentration shift-keying (MCSK), all in binary forms, has been analyzed based on Poisson modeling. In its analysis for CSK and MoSK, a current symbol is assumed only to experience ISI from one previously sent symbol, while for MCSK it is assumed that there is 
no ISI. Poisson modeling has also been introduced in [27, 30] for deriving the probability density function (PDF) and cumulative density function (CDF) of the DMC systems with CSK modulation. In $[17,31]$, the authors have analyzed the false-alarm probability, detection probability, and ultimately the error probability of the DMC systems with binary and $M$ ary CSK modulations, when the counting noise and ISI are both modeled as Gaussian noise. In [32], the authors have analyzed the error performance of the DMC systems with pulse-based modulation, and energy or amplitude detection, and some closed-form expressions have been derived also by approximating both the counting noise and ISI as Gaussian noise. In [33], the authors have analyzed the error performance (also capacity) of the DMC systems employing an $M$-ary amplitude modulation or an extended modulation scheme. In their analysis, molecules transmitted associated with different symbols are assumed to arrive at the receiver according to the binomial distributions, which are then approximated by the Gaussian distributions for deriving the error probability. Very recently, the authors of [34] have carried out the analysis for the DMC systems with both CSK and MoSK modulations, when modeling the number of molecules hitting the receiver at any time as the binomial distribution.

Against the background, in this paper, we focus on the bit error rate (BER) analysis of the DMC systems with binary CSK modulation, which we refer to as the on-off keying (OOK) modulation. According to the results established in [35] as well as the analysis and theory in [27,36], when the number of molecules appearing in a DMC environment is large, the number of molecules within an observation space can be accurately modelled by the Poisson distribution. Hence, based on the Poisson modeling, we first derive an exact BER expression under the Poisson modeling. In our analysis, both counting noise and ISI are considered, and furthermore, we assume that the length of ISI is arbitrary, and varies according to the transmission distance, data rate, etc. However, it shows that the BER expression derived from the Poisson modeling in some cases may be too complicated to evaluate, which is mainly the responsibility of two aspects. The first one is due to the discrete Poisson distribution, while the second one is resulted from the long ISI. In order to cope with the first aspect, we consider two continuous distributions for approximating the Poisson distribution, which are the Gaussian- and Gamma-approximation. Based on these two approximation approaches, we then derive the corresponding closed-form BER expressions. In order to mitigate the computational complexity resulted from long ISI, we also propose two approaches. The first one is the Monte-Carlo approach, which computes the BER by randomly generating a sufficient number of binary bits imposing ISI, instead of considering all the possible sequences that may be extreme in practice. In the second approach, we divide the ISI into two components. The first ISI component is generated by a number of bits sent in the front of the reference bit being detected, and all the possible combinations of these bits are considered in the BER computation. By contrast, the second ISI component consists of all the bits other than those included in the first component. The ISI imposed by these bits is approximated as a Poisson (or Gaussian, etc.) random variable that is independent of the specific bit sequence. Both the above approaches are then integrated with the Poisson, Gaussian- and Gamma-approximation approaches, and correspondingly, the formulas for BER evaluation are provided. Following the BER analysis of the DMC systems with OOK modulation, we then analyze the BER of the OOK-modulated DMC systems with ISI cancellation (ISIC) [1,26]. In our analysis, the Poisson modeling, Gaussian-approximation, and the two approaches for ISI handling are considered. Correspondingly, the closedform BER expressions are derived. Finally, our analytical approaches are compared and validated by a range of numerical and simulation results. The BER performance of the OOK-modulated DMC systems without/with ISIC is studied and compared by considering the impact of different system parameters. The accuracy of various approximation approaches is demonstrated via comparison of the BER evaluated from the analytical expressions and/or obtained by simulations. Our studies and performance results demonstrate that all the BER expressions derived are valid for predicting the BER of the OOK-modulated DMC systems without/with ISIC. Furthermore, in some communication scenarios, employing the lowcomplexity ISIC scheme is capable of improving the error performance of DMC systems.

We should note that, although in this paper we consider only the OOK modulation, the analytical approaches considered in Sections III and IV may be extended straightforwardly to the DMC systems with other types of modulation schemes, including $M$-ary CSK [10-18], MoSK [3, 14, 15, 20], etc.

The rest of the paper is organized as follows. In section II, we describe the DMC system model and state the main assumptions. Section III analyzes the BER of the DMC systems employing OOK modulation, while Section IV deals with the BER of the OOK-modulated DMC systems with ISIC. In Section V, we demonstrate the BER performance results obtained from numerical computation and particle-based as well as Monte-Carlo simulations. Finally, in Section VI, we summarize the main observations obtained from the studies.

\section{System MOdel AND Assumptions}

We consider a typical DMC system [13,25-27], which consists of a transmitter, a molecular diffusion channel and a receiver. At the transmitter, information is emitted via molecular release patterns, which propagate over a molecular diffusion channel. The receiver is a passive observer [30], which recovers information according to the time-varying molecule concentration measured within a detection space. For convenience of study, some typical assumptions generally used in references, such as, [13,26], are applied. In detail, the transmitter is assumed to be a point molecule source with released molecules not interacting with the transmitter. The receiver is assumed to be able to ideally measure the molecule concentration within a spherical detection space with a radius of $\rho$. We assume that $\rho$ is small with respect to the communication distance, yielding a near-uniform concentration within the detection space. In other words, the receiver is idealized as a point receiver. During a transmission, 


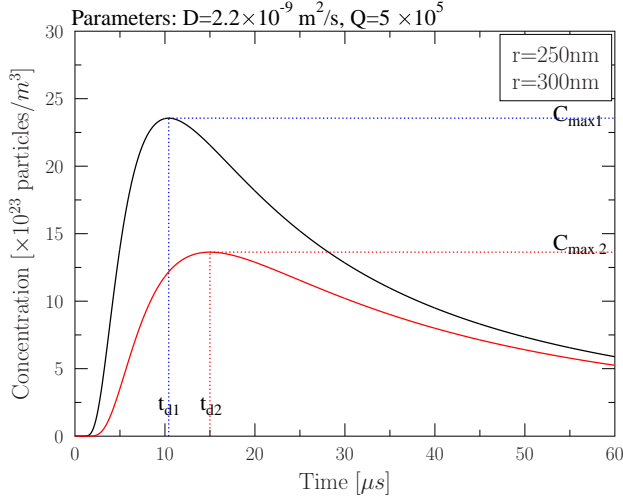

Fig. 1. Pulse functions of molecule concentration with respect time and distance.

the positions of both transmitter and receiver are assumed at fixed locations. Furthermore, we assume that molecules at transmitter are released as impulses of molecules. Based on these assumptions, then, according to the Fick's law of diffusion [13], when an impulse of $Q$ molecules are released by a transmitter at $t=0$, the molecule concentration measured at a point having a distance $r$ from the transmitter can be expressed as $[13,26]$

$$
c(t)=\frac{Q}{(4 \pi D t)^{\frac{3}{2}}} \exp \left(-\frac{r^{2}}{4 D t}\right), t>0
$$

where $D$ is medium's diffusion coefficient, and $t>0$ is the observation time at receiver.

The molecule concentration $c(t)$ is a time-domain pulse function, having a shape as shown in Fig. 1, when assuming that an impulse of molecules is released at $t=0$. It can be shown [13] that the concentration at a point having a fixed distance $r$ from the transmitter reaches its maximum at the instant $t_{d}=\frac{r^{2}}{6 D_{3}}$, and the peak value of $c(t)$ is $c_{\max }=c_{\max }(r)=\left(\frac{3}{2 \pi e}\right)^{\frac{3}{2}} \frac{Q}{r^{3}}$.

\section{A. DMC Employing On-Off Keying Modulation}

DMC with OOK modulation has been investigated in many references, such as in $[13,15,25-27]$. Its principle can be briefly described as follows. Let $\left\{b_{j}\right\}=\left\{b_{0}, b_{1}, \ldots, b_{j}, \ldots\right\}$ be a binary information sequence, where $b_{j} \in\{0,1\}$. When the OOK modulation is employed, the transmitter emits an impulse of molecules to send $b_{j}=1$, but releases no molecules for sending $b_{j}=0$. Therefore, after sending the $u$ th bit, the molecule concentration measured by the receiver within its detection space can be expressed as [25]

$$
z(t)=\sum_{j=0}^{u} b_{j}\left[c(t-j T)+n_{j}(t)\right], u T \leq t<(u+1) T
$$

where $T$ represents the symbol interval, which is also the bit interval when binary OOK modulation is considered. Hence, the information rate is $R=1 / T$ bits per second (bps). In (2), $u=\lceil t / T\rceil$ is the largest integer not exceeding $t / T$, with $(u+1)$ representing the total number of bits transmitted within $[0, t)$. Finally, $n_{j}(t)$ in (2) is due to the particle counting noise associated with the process of sending $b_{j}$. According to [35], counting noise is the result of the (random) Brownian motions of molecules, which cause an unwanted perturbation to the concentration predicted by the Fick's diffusion law.

As shown in Fig. 1, when an impulse of molecules is sent at $t=0$, it would be desirable for the receiver to sample at $t=t_{d}$ for the maximum molecule concentration, in order to make most reliable detection. Therefore, in order to detect the $u$ th bit, we assume that the receiver samples for the molecule concentration at $t=u T+\hat{t}_{d}$, where $\hat{t}_{d}$ represents the estimate to $t_{d}$. This gives the decision variable for detection of the $u$ th bit as

$$
\begin{aligned}
Z_{u} & =z\left(t=u T+\hat{t}_{d}\right) \\
& =\sum_{j=0}^{u} b_{j}\left[c\left([u-j] T+\hat{t}_{d}\right)+n_{j}\left(u T+\hat{t}_{d}\right)\right], \quad u=0,1, \ldots
\end{aligned}
$$

Let us define $c_{u-j}=c\left([u-j] T+\hat{t}_{d}\right)$ and $n_{j u}=n_{j}\left(u T+\hat{t}_{d}\right)$. Then, the above equation can be rewritten as

$$
Z_{u}=\sum_{j=0}^{u} b_{j}\left[c_{u-j}+n_{j u}\right], \quad u=0,1, \ldots
$$

Explicitly, the detection of bit $u$ experiences ISI. Let us assume that the maximum length of ISI is $L$ bits. Then, upon applying the variable transform of $i=u-j$, we can express $Z_{u}$ of (4) in the form of

$$
\begin{aligned}
Z_{u} & =\sum_{\substack{j=\max \{0, u-L\} \\
\min \{L, u\}}}^{u} b_{j}\left[c_{u-j}+n_{j u}\right] \\
& =\sum_{i=0}^{\operatorname{mos}} b_{u-i}\left[c_{i}+n_{(u-i) u}\right], \quad u=0,1, \ldots
\end{aligned}
$$

where $c_{i}=c\left(i T+\hat{t}_{d}\right)$. Furthermore, for convenience, we may also write (5) as

$$
Z_{u}=\sum_{i=0}^{\min \{L, u\}} b_{u-i} c_{i}+n_{u}, \quad u=0,1, \ldots
$$

where $n_{u}=\sum_{i=0}^{\min \{L, u\}} b_{u-i} n_{(u-i) u}$.

Let $V_{R}=\frac{4}{3} \pi \rho^{3}$ be the volume of the spherical detection space. Then, as given in [25,27,35], $Y_{u}=V_{R} Z_{u}$ can be accurately modeled as a Poisson distributed random variable with both the mean and variance given by $\mu_{Y}=$ $V_{R} \sum_{i=0}^{\min \{L, u\}} b_{u-i} c_{i}$, which is expressed as $Y_{u} \sim \mathcal{P}\left(\mu_{Y}\right)$. Furthermore, when $\mu_{Y}$ is sufficiently large, making the Gaussian approximation applicable, $Y_{u}$ can be approximated as a Gaussian random variable with both the mean and the variance given by $\mu_{Y}$, which is expressed as $Y_{u} \sim \mathcal{N}\left(\mu_{Y}, \mu_{Y}\right)$. Correspondingly, $Z_{u}$ in (6) obeys the Gaussian distribution of $Z_{u} \sim \mathcal{N}\left(\mu_{Z}, \sigma_{Z}^{2}\right)$ [26], where the mean and variance are given by $\mu_{Z}=\sum_{i=0}^{\min \{L, u\}} b_{u-i} c_{i}$ and $\sigma_{Z}^{2}=V_{R}^{-1} \sum_{i=0}^{\min \{L, u\}} b_{u-i} c_{i}$. Hence, the PDF of the noise samples $\left\{n_{u}\right\}$ seen in (6) is $n_{u} \sim \mathcal{N}\left(0, \sigma_{Z}^{2}\right)$.

Let $C_{T}$ be a threshold used by the OOK modulation for decision making. Considering that the concentration presenting at the receiver has a function as shown in Fig. 1, it is 
sometimes convenient to set the threshold relative to the peak $c_{\max }$ as

$$
C_{T}=\alpha c_{\max }
$$

where $0 \leq \alpha \leq 1$ can be referred to as the normalized threshold. Consequently, the receiver makes the decision for a bit by comparing the measured concentration with the threshold according to the rules of

$$
\hat{b}_{u}= \begin{cases}1, & \text { when } Y_{u}>V_{R} C_{T}, Z_{u}>C_{T}, \text { or } \frac{Z_{u}}{C_{\text {max }}}>\alpha \\ 0, & \text { when } Y_{u} \leq V_{R} C_{T}, Z_{u} \leq C_{T}, \text { or } \frac{Z_{u}}{C_{\max }} \leq \alpha\end{cases}
$$

Here we should note that $Y_{u}$ represents the number of molecules counted within the detection space with a volume of $V_{R}$, hence it is an integer.

As seen, e.g., in (5), the detection of $b_{u}$ experiences ISI, which becomes severer as the data rate becomes higher, resulting in a reduced symbol duration. Without ISI mitigation, the performance of DMC may be severely degraded. Below we consider the ISI mitigation in the OOK-modulated DMC systems.

\section{B. Inter-Symbol Interference Cancellation}

In [26], a simple ISIC method has been proposed, when the DMC with OOK modulation is assumed. In this method, the receiver forms decision variables as

$$
\begin{aligned}
Z_{u}= & z\left(t=u T+\hat{t}_{d}\right)-z(t=u T) \\
= & \sum_{j=0}^{u} b_{j}\left[c\left([u-j] T+\hat{t}_{d}\right)+n_{j}\left(u T+\hat{t}_{d}\right)\right] \\
& -\sum_{j=0}^{u} b_{j}\left[c([u-j] T)+n_{j}(u T)\right], \quad u=0,1, \ldots
\end{aligned}
$$

which is the difference between the two observations, $z(t=$ $\left.u T+\hat{t}_{d}\right)$ and $z(t=u T)$, obtained at $t=u T+\hat{t}_{d}$ and $t=u T$, respectively. With the aid of Fig. 1, we can know that the first observation measures the molecule concentration peak after sending bit $b_{u}$. By contrast, the second observation obtained at the start of sending $b_{u}$ provides a reference for the ISI imposed on $b_{u}$ at $t=u T+\hat{t}_{d}$. Therefore, after the subtraction operation as seen in (9) and (10), most of the ISI will be cancelled. From Fig. 1 we can be implied that this approach is in particular effective, when the transmission rate $R=1 / T$ is relatively low, resulting in that the ISI measured at $t=u T$ is nearly the same as that measured at $t=u T+\hat{t}_{d}$.

However, when the transmission rate becomes high, the ISI obtained at $t=u T$ may be very different from that at $t=$ $u T+\hat{t}_{d}$, as implied by Fig. 1. This becomes even severer, when $b_{u-1}=+1$ is sent before $b_{u}$. Let us rearrange (10) to express the decision variable as

$$
\begin{aligned}
Z_{u}= & b_{u} c\left(\hat{t}_{d}\right)+\sum_{j=0}^{u-1} b_{j}\left[c\left([u-j] T+\hat{t}_{d}\right)-c([u-j] T)\right] \\
& +\sum_{j=0}^{u} b_{j}\left[n_{j}\left(u T+\hat{t}_{d}\right)-n_{j}(u T)\right], \quad u=0,1, \ldots
\end{aligned}
$$

where $c(0)=0$ is assumed. Furthermore, we also have $n_{u}(u T)=0$, as it is the counting noise contributed by the $u$ th bit that has not yet sent at $t=u T$. At the righthand side (RHS) of (11), the first term is the desired observation, the second term is the residue ISI due to non-ideal ISIC, while the last term is the counting noise presenting at $t=u T+\hat{t}_{d}$ and at $t=u T$. Assume that the transmission rate is high, resulting in that the symbol duration $T$ is only slightly larger than $t_{d}$. Then, with the aid of Fig. 1, we can be implied that the ISI at $t=u T$ can be much larger than the ISI at $t=u T+\hat{t}_{d}$, due to the fast decrease of molecule concentration resulted from the impulses of molecules sent most recently. In this case, the ISIC based on (9) results in over cancellation, yielding a large residue ISI given by the second term at the RHS of (11). Furthermore, as seen in (11), the counting noise after the ISIC is contributed by both the counting noise presenting at $t=u T$ and that at $t=u T+\hat{t}_{d}$. According to [35], provided that $\hat{t}_{d}>\rho^{2} / D$, two adjacent samples of the counting noise process can be regarded as statistically independent. In this case, the ISIC operation of (9) enhances the noise, which may further degrade the achievable performance of DMC.

In order to improve the performance of the ISIC, a more accurate estimate to the ISI at $t=u T+\hat{t}_{d}$ is desirable, while simultaneously mitigating the impact from noise enhancement. With this motivation, in [1], we have proposed an improved ISIC scheme, which forms the decision variable according to the formula

$$
Z_{u}=z\left(t=u T+\hat{t}_{d}\right)-\lambda_{u} z(t=u T)
$$

where $0 \leq \lambda_{u} \leq 1$ is used to scale the ISI measured at $t=u T$. Following (11), we can express (12) in detail as

$$
\begin{aligned}
Z_{u}= & b_{u} c\left(\hat{t}_{d}\right)+\sum_{j=0}^{u-1} b_{j}\left[c\left([u-j] T+\hat{t}_{d}\right)-\lambda_{u} c([u-j] T)\right] \\
& +\sum_{j=0}^{u} b_{j}\left[n_{j}\left(u T+\hat{t}_{d}\right)-\lambda_{u} n_{j}(u T)\right], \quad u=0,1, \ldots
\end{aligned}
$$

Explicitly, at the RHS of (13), the desired term is not affected by the ISIC, while the noise enhancement in the third term is mitigated due to $\lambda_{u}<1$.

Furthermore, in [1], we have proposed the approaches for estimating the scaling factor in (12). For completeness, these approaches are also detailed below. First, the optimum value of $\lambda_{u}$ for given transmission rate and pulse shape may be obtained via numerical/simulation approaches. Second, we may estimate the scaling factor with the motivation to fully cancel the ISI presenting at $t=u T+\hat{t}_{d}$ as follows. Let the average ISI at $t$ be expressed as $I_{S I}(t)$. Then, we can find an estimate to $\lambda_{u}$ by solving the equation of

$$
\lambda_{u} I_{S I}(u T)=I_{S I}\left(u T+\hat{t}_{d}\right)
$$

where both $I_{S I}(u T)$ and $I_{S I}\left(u T+\hat{t}_{d}\right)$ can be calculated, once we know the correct estimates of $\hat{b}_{0}, \hat{b}_{1}, \ldots, \hat{b}_{u-1}$ for the transmitted bits $b_{0}, b_{1}, \ldots, b_{u-1}$. However, considering all the past symbols results in high complexity and possibly degraded performance due to error propagation and noise 
enhancement, as above-mentioned. Furthermore, in practice, it may not be necessary to consider all the past symbols, as the ISI contributed by a pulse becomes nearly flat after some time, as shown in Fig. 1. When taking all these into account, we may estimate $\lambda_{u}$ as follows. Let us consider $J(J<u)$ most recent bits. Then, we can compute $J$ coefficients corresponding to the most recent $J$ bits as

$$
\beta_{u-1}=\frac{c\left(T+\hat{t}_{d}\right)}{c(T)}, \ldots, \beta_{u-J}=\frac{c\left(J T+\hat{t}_{d}\right)}{c(J T)}
$$

if the pulse $c(t)$ presenting at the receiver is known. The value of $J$ can be chosen, so that the remaining ISI at $u T$ and that at $u T+\hat{t}_{d}$ satisfy

$$
C_{J}(u)=\sum_{j=0}^{J-1} b_{j} c\left([u-j] T+\hat{t}_{d}\right) \approx \sum_{j=0}^{J-1} b_{j} c([u-j] T)
$$

which can be evaluated as

$$
C_{J}(u)=z(t=u T)-\sum_{l=1}^{J} \hat{b}_{u-l} c(l t)
$$

Alternatively, we may simply use the average value of $C_{J}(u)$, which can be estimated as

$$
\bar{C}_{J}=\frac{1}{N} \sum_{u=J+1}^{J+N} C_{J}(u)
$$

Finally, with the aid of (15) and (16), as well as the estimated bit values, (14) can be expressed as

$\hat{\lambda}_{u}\left(\sum_{j=1}^{J} \hat{b}_{u-j} c(j T)+C_{J}(u)\right)=\sum_{j=1}^{J} \hat{b}_{u-j} \beta_{u-j} c(j T)+C_{J}(u)$

from which $\lambda_{u}$ can be expressed as

$$
\hat{\lambda}_{u}=\frac{\sum_{j=1}^{J} \hat{b}_{u-j} \beta_{u-j} c(j T)+C_{J}(u)}{\left(\sum_{j=1}^{J} \hat{b}_{u-j} c(j T)+C_{J}(u)\right)}
$$

In the above equation, if $C_{J}(u) \approx 0$, we have

$$
\hat{\lambda}_{u}=\frac{\sum_{j=1}^{J} \hat{b}_{u-j} \beta_{u-j} c(j T)}{\sum_{j=1}^{J} \hat{b}_{u-j} c(j T)}
$$

According to above analysis, if detection is highly reliable, we may expect that the ISIC seen in (12) (and (13)) is fully removed or significantly reduced. If the detection becomes less reliable, the ISIC results in error propagation. As seen in (21), $\hat{\lambda}_{u}$ is dependent on the $J$ bits received before the $u$ th bit. If a bit is detected in error, it will affect the detection of its following $J$ bits. In order to mitigate the error propagation, in practice, the value of $\lambda_{u}$ in (12) may be adjusted according to the detection reliability. When the detection reliability is low, a $\lambda_{u}$ smaller than $\hat{\lambda}_{u}$ of (21) may be employed, in order to mitigate the effect of error propagation. By contrast, when the detection reliability increases, the value of $\lambda_{u}$ can be gradually increased towards the value of $\hat{\lambda}_{u}$ estimated by (21), in order to further enhance the detection reliability. However, we should note that the ISIC results in additional noise, which increases as $\lambda_{u}$ increases. Therefore, for a given $\lambda_{u}$, there exists a tradeoff between ISIC and noise enhancement.

In the following two sections, we will analyze the bit error rate (BER) of the DMC systems employing or without employing the ISIC. Specifically in Section III, we address the BER performance of the DMC systems employing OOK modulation, while in Section IV, the BER performance of the OOK-modulated DMC systems with ISIC is analyzed.

\section{BER ANALYSIS OF DMC SYSTEMS EMPLOYING OOK MODULATION}

We analyze the BER of the DMC systems employing the OOK modulation based on the decision variable given by (5). In our analysis, we assume that the DMC process is in its static state, meaning that $u>>L$, here $L$ is the maximum length of ISI. Correspondingly, the decision variable of (5) can be written as

$$
Z_{u}=\sum_{i=0}^{L} b_{u-i}\left[c_{i}+n_{(u-i) u}\right]
$$

Furthermore, we assume that the sampling duration, which is the bit duration $T$, satisfies the condition of $T>\rho^{2} / D$ [35]. Hence, adjacent noise samples are independent. Additionally, we assume that the transmitted binary bits obey the distribution of $P(1)=P(0)=0.5$.

\section{A. Poisson Approach: Exact BER Analysis}

Let us first analyze the exact BER of the DMC systems employing OOK modulation based on the Poisson distribution of $Y_{u}=V_{R} Z_{u}$, which for convenience is referred to as the Poisson approach. Note that in literature, the Poisson approach has been employed by a number of references, e.g., [21,27, 30], for performance studies, as detailed in the literature review in Section I. As shown in Section II-A, the decision variable $Y_{u}$ obeys the Poisson distribution with the probability mass function (PMF) given by $\mathcal{P}\left(\mu_{Y}\right)$, where $\mu_{Y}=V_{R} \sum_{i=0}^{L} b_{u-i} c_{i}$. Explicitly, $\mu_{Y}$ is dependent on the transmitted data sequence. Let us define a vector $\boldsymbol{b}_{L}=\left[b_{u-L}, \ldots, b_{u-1}\right]^{T}$ containing the $L$ bits imposing ISI on $b_{u}$. Then, when $b_{u}=1$, we express the Poisson PMF as [36]

$$
f_{Y_{u}}\left(n \mid \boldsymbol{b}_{L}, b_{u}=1\right)=\frac{\mu_{Y_{1}}^{n} e^{-\mu_{Y_{1}}}}{n !}, n=0,1,2, \ldots
$$

where $\mu_{Y_{1}}=V_{R}\left(c_{0}+\sum_{i=1}^{L} b_{u-i} c_{i}\right)$. When $b_{u}=0$, the Poisson PMF can be expressed as

$$
f_{Y_{u}}\left(n \mid \boldsymbol{b}_{L}, b_{u}=0\right)=\frac{\mu_{Y_{0}}^{n} e^{-\mu_{Y_{0}}}}{n !}, n=0,1,2, \ldots
$$

associated with $\mu_{Y_{0}}=V_{R} \sum_{i=1}^{L} b_{u-i} c_{i}$. The BER of the DMC systems employing the OOK modulation is given by

$$
\begin{aligned}
P_{b} & =\frac{1}{2} P\left(\hat{b}_{u}=0 \mid b_{u}=1\right)+\frac{1}{2} P\left(\hat{b}_{u}=1 \mid b_{u}=0\right) \\
& =\frac{1}{2}\left(P_{M}+P_{F A}\right)
\end{aligned}
$$

where $P_{M}=P\left(\hat{b}_{u}=0 \mid b_{u}=1\right)$ is the probability of detecting $\hat{b}_{u}=0$ for an actually transmitted $b_{u}=1$, which is usually 
referred to as the miss-probability, while $P_{F A}=P\left(\hat{b}_{u}=\right.$ $\left.1 \mid b_{u}=0\right)$ is the probability of detecting $\hat{b}_{u}=1$ for an actually transmitted $b_{u}=0$, which is called as the false-alarm probability. Let us below derive these probabilities in detail.

First, given a detection threshold $C_{T}$ with respect to the molecule concentration, the miss-probability can be expressed as

$$
\begin{aligned}
P_{M} & =P\left(\hat{b}_{u}=0 \mid b_{u}=1\right) \\
& =P\left(Y_{u} \leq V_{R} C_{T} \mid b_{u}=1\right) \\
& =\sum_{\boldsymbol{b}_{L} \in \mathcal{B}^{L}} P\left(\boldsymbol{b}_{L}\right) P\left(Y_{u} \leq V_{R} C_{T} \mid \boldsymbol{b}_{L}, b_{u}=1\right)
\end{aligned}
$$

where $\mathcal{B}=\{1,0\}, P\left(\boldsymbol{b}_{L}\right)$ is the probability of the occurrence of a specific sequence of $\boldsymbol{b}_{L}$. When assuming that $P(1)=P(0)=0.5$, we have $P\left(\boldsymbol{b}_{L}\right)=1 / 2^{L}$. Finally, $P\left(Y_{u} \leq V_{R} C_{T} \mid \boldsymbol{b}_{L}, b_{u}=1\right)$ is the probability conditioned on a given data sequence of $\boldsymbol{b}_{L}$ and $b_{u}=1$. With the aid of the PMF given by (23), we have [36]

$$
P\left(Y_{u} \leq V_{R} C_{T} \mid \boldsymbol{b}_{L}, b_{u}=1\right)=\sum_{n=0}^{\left\lceil V_{R} C_{T}\right\rceil} \frac{\mu_{Y_{1}}^{n} e^{-\mu_{Y_{1}}}}{n !}
$$

Upon substituting this result into (26), we obtain the missprobability of

$$
P_{M}=\frac{1}{2^{L}} \sum_{\boldsymbol{b}_{L} \in \mathcal{B}^{L}} \sum_{n=0}^{\left\lceil V_{R} C_{T}\right\rceil} \frac{\mu_{Y_{1}}^{n} e^{-\mu_{Y_{1}}}}{n !}
$$

For the false-alarm probability, we have the relationships of

$$
\begin{aligned}
P_{F A} & =P\left(\hat{b}_{u}=1 \mid b_{u}=0\right) \\
& =P\left(Y_{u}>V_{R} C_{T} \mid b_{u}=0\right) \\
& =1-P\left(Y_{u} \leq V_{R} C_{T} \mid b_{u}=0\right) \\
& =1-\sum_{\boldsymbol{b}_{L} \in \mathcal{B}^{L}} P\left(\boldsymbol{b}_{L}\right) P\left(Y_{u} \leq V_{R} C_{T} \mid \boldsymbol{b}_{L}, b_{u}=0\right)
\end{aligned}
$$

Similar to (27), we can obtain

$$
P_{F A}=1-\frac{1}{2^{L}} \sum_{\boldsymbol{b}_{L} \in \mathcal{B}^{L}} \sum_{n=0}^{\left\lceil V_{R} C_{T}\right\rceil} \frac{\mu_{Y_{0}}^{n} e^{-\mu_{Y_{0}}}}{n !}
$$

Finally, applying both (28) and (30) into (25), the BER of the DMC systems employing OOK modulation can be expressed as

$$
P_{b}=\frac{1}{2}+\frac{1}{2^{L+1}} \sum_{\boldsymbol{b}_{L} \in \mathcal{B}^{L}}\left(\sum_{n=0}^{\left\lceil V_{R} C_{T}\right\rceil} \frac{\mu_{Y_{1}}^{n} e^{-\mu_{Y_{1}}}-\mu_{Y_{0}}^{n} e^{-\mu_{Y_{0}}}}{n !}\right)
$$

\section{B. Gaussian-Approximation}

From (31), we can see that evaluating the BER has the complexity of $\mathcal{O}\left(\left\lceil V_{R} C_{T}\right\rceil 2^{L}\right)$ contributed by the two sum in the formula, where $2^{L}$ is due to the $L$-length binary data sequence imposing ISI, while $\left\lceil V_{R} C_{T}\right\rceil$ is the result of the discrete Poisson distribution. If the discrete Poisson distribution can be approximated by a continuous distribution, the second sum in (31) can be replaced by an integration, which may be simplified to obtain a closed-form convenient for evaluation.

It is well-known that the Poisson distribution has the property [36] that it can be approximated by a Gaussian distribution, if the Poisson parameter $(\mu)$ is sufficiently large, e.g., $\mu>20$. Therefore, in literature, the performance of some DMC systems has been studied under the Gaussian modeling of counting noise [17,27,31,32]. In our case, if $\mu_{Y_{1}}$ in (23) and $\mu_{Y_{0}}$ in (24) are sufficiently large, then both $f_{Y_{u}}\left(n \mid \boldsymbol{b}_{L}, b_{u}=1\right)$ and $f_{Y_{u}}\left(n \mid \boldsymbol{b}_{L}, b_{u}=1\right)$ can be approximated by the Gaussian distributions. In this case, we can derive the BER directly from the concentration-based decision variable $Z_{u}$ given by (22). Therefore, when assuming $u>>L$, the PDFs of $Z_{u}$ conditioned on $\boldsymbol{b}_{L}, b_{u}=1$ or 0 can be expressed as [36]

$$
\begin{aligned}
& f_{Z_{u}}\left(y \mid \boldsymbol{b}_{L}, b_{u}=1\right)=\frac{1}{\sqrt{2 \pi} \sigma_{Z_{1}}} \exp \left(-\frac{\left[y-\mu_{Z_{1}}\right]^{2}}{2 \sigma_{Z_{1}}^{2}}\right), \\
& f_{Z_{u}}\left(y \mid \boldsymbol{b}_{L}, b_{u}=0\right)=\frac{1}{\sqrt{2 \pi} \sigma_{Z_{0}}} \exp \left(-\frac{\left[y-\mu_{Z_{0}}\right]^{2}}{2 \sigma_{Z_{0}}^{2}}\right)
\end{aligned}
$$

where $-\infty<y<\infty$, by definition, we have

$$
\begin{aligned}
& \mu_{Z_{1}}=c_{0}+\sum_{i=1}^{L} b_{u-i} c_{i} ; \quad \sigma_{Z_{1}}^{2}=\frac{c_{0}}{V_{R}}+\frac{1}{V_{R}} \sum_{i=1}^{L} b_{u-i} c_{i} \\
& \mu_{Z_{0}}=\sum_{i=1}^{L} b_{u-i} c_{i} ; \quad \sigma_{Z_{0}}^{2}=\frac{1}{V_{R}} \sum_{i=1}^{L} b_{u-i} c_{i}
\end{aligned}
$$

which are all functions of $\boldsymbol{b}_{L}$. Therefore, following the analysis in (26), the miss-probability $P_{M}$ can be expressed as

$$
\begin{aligned}
P_{M} & =\frac{1}{2^{L}} \sum_{\boldsymbol{b}_{L} \in \mathcal{B}^{L}} P\left(Z_{u} \leq C_{T} \mid \boldsymbol{b}_{L}, b_{u}=1\right) \\
& =1-\frac{1}{2^{L}} \sum_{\boldsymbol{b}_{L} \in \mathcal{B}^{L}} P\left(Z_{u}>C_{T} \mid \boldsymbol{b}_{L}, b_{u}=1\right) \\
& =1-\frac{1}{2^{L}} \sum_{\boldsymbol{b}_{L} \in \mathcal{B}^{L}} \frac{1}{\sqrt{2 \pi} \sigma_{Z_{1}}} \int_{C_{T}}^{\infty} \exp \left(-\frac{\left[y-\mu_{Z_{1}}\right]^{2}}{2 \sigma_{Z_{1}}^{2}}\right) d y \\
& =1-\frac{1}{2^{L}} \sum_{\boldsymbol{b}_{L} \in \mathcal{B}^{L}} Q\left(\frac{C_{T}-\mu_{Z_{1}}}{\sigma_{Z_{1}}}\right)
\end{aligned}
$$

where $Q(x)$ is the Gaussian Q-function [37] defined as $Q(x)=(2 \pi)^{-1 / 2} \int_{x}^{\infty} e^{-t^{2} / 2} d t$, which is the built-in function of many software packages, e.g., Matlab, for numerical computation.

Similarly, the false-alarm probability $P_{F A}$ can be derived as

$$
\begin{aligned}
P_{F A} & =\frac{1}{2^{L}} \sum_{\boldsymbol{b}_{L} \in \mathcal{B}^{L}} P\left(Z_{u}>C_{T} \mid \boldsymbol{b}_{L}, b_{u}=0\right) \\
& =\frac{1}{2^{L}} \sum_{\boldsymbol{b}_{L} \in \mathcal{B}^{L}} \frac{1}{\sqrt{2 \pi} \sigma_{Z_{0}}} \int_{C_{T}}^{\infty} \exp \left(-\frac{\left[y-\mu_{Z_{0}}\right]^{2}}{2 \sigma_{Z_{0}}^{2}}\right) d y \\
& =\frac{1}{2^{L}} \sum_{\boldsymbol{b}_{L} \in \mathcal{B}^{L}} Q\left(\frac{C_{T}-\mu_{Z_{0}}}{\sigma_{Z_{0}}}\right)
\end{aligned}
$$

Finally, when substituting both (34) and (35) into (25), the BER of the OOK modulated DMC systems under the Gaussian-approximation can be obtained. 


\section{Gamma-Approximation}

In comparison to (31), the BER expression obtained from the Gaussian-approximation may be slightly advantageous for evaluation. However, in order to apply the Gaussianapproximation, the mean $\mu_{Y_{1}}=V_{R}\left(c_{0}+\sum_{i=1}^{L} b_{u-i} c_{i}\right)$ and $\mu_{Y_{0}}=V_{R}\left(\sum_{i=1}^{L} b_{u-i} c_{i}\right)$ need to be relatively large, typically, exceed 20 . Since $V_{R}$ is usually a very small value in comparison to the diffusion space of molecules, the above conditions may not be satisfied, unless the number of molecules $Q$ per transmission impulse is very big. This situation often means that the signal-to-noise ratio (SNR) for DMC is high. In a nutshell, the Gaussian-approximation is only suitable for high SNR scenarios. Furthermore, as seen in (32), the Gaussian distributions are defined in the range of $(-\infty, \infty)$, which makes the Gaussian-approximation for the Poisson distribution defined in $(0, \infty)$ insensible, especially when the mean of $\mu_{Y_{1}}$ or $\mu_{Y_{0}}$ is relatively small. Considering the above issues, in this section, we propose the Gamma-approximation [38] to approximate the Poisson distribution as a Gamma-distribution with the PDF in the form of [39]

$$
f_{Y}(y)=\frac{1}{\Gamma(m) \Omega^{m}} y^{m-1} \exp \left(-\frac{y}{\Omega}\right), 0 \leq y<\infty
$$

where $m$ is the shape parameter and $\Omega$ is the scale parameter, while $\Gamma(x)$ is the gamma function. It can be shown that the Gamma distributed variable $Y$ has the mean and variance given by [39]

$$
E[Y]=m \Omega, \quad \operatorname{Var}[Y]=m \Omega^{2}
$$

From these relationship, we can readily know that

$$
m=\frac{E^{2}[Y]}{\operatorname{Var}[Y]}, \quad \Omega=\frac{\operatorname{Var}[Y]}{E[Y]}
$$

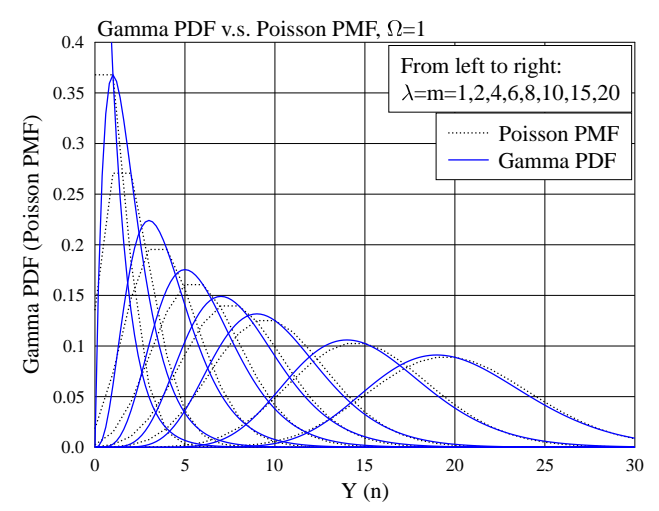

Fig. 2. Comparison between the Poisson PMF and the corresponding Gamma PDF.

Assume a Poisson PMF of $f(n \mid \lambda)=\lambda^{n} e^{-\lambda} / n$ !. Then, it can be shown that for the corresponding Gamma PDF of (36), we have $m=\lambda$ and $\Omega=1$. The comparison between the Poisson PMF and Gamma PDF are shown in Figs. 2 for some $\lambda, m$ values and $\Omega=1$. Explicitly, the Gamma distribution is capable of providing a good approximation for the Poisson distribution for all the considered $\lambda$ values, and more closer approximation presents, as the value of $\lambda$ increases. In fact, from the research of radio communication channel modeling [40-43], the Gamma distribution (or the Nakagami distribution for the square-roots of Gamma distributed random variables) usually fits well the measurement data in terms of SNR (or magnitude) from practical experiments. This might imply that the Nakagami and Gamma distributions are also the good tools for describing the statistical properties of DMC channels, especially in the case when continuous distribution functions are required.

Let us now return to derive the BER of the OOK-modulated DMC systems under the Gamma-approximation. In this case, we can express the Gamma PDFs conditioned on $b_{u}=1$ and $b_{u}=0$ respectively as

$$
\begin{aligned}
& f_{Z_{u}}\left(y \mid \boldsymbol{b}_{L}, b_{u}=1\right)=\frac{1}{\Gamma\left(m_{1}\right) \Omega_{1}^{m_{1}}} y^{m_{1}-1} \exp \left(-\frac{y}{\Omega_{1}}\right), \\
& f_{Z_{u}}\left(y \mid \boldsymbol{b}_{L}, b_{u}=0\right)=\frac{1}{\Gamma\left(m_{0}\right) \Omega_{0}^{m_{0}}} y^{m_{0}-1} \exp \left(-\frac{y}{\Omega_{0}}\right)
\end{aligned}
$$

where $0 \leq y<\infty$. With the aid of (33), the shape and scale parameters in (39) are given by

$$
\begin{aligned}
& m_{1}=\frac{\mu_{Z_{1}}^{2}}{\sigma_{Z_{1}}^{2}}, \quad \Omega_{1}=\frac{\sigma_{Z_{1}}^{2}}{\mu_{Z_{1}}} \\
& m_{0}=\frac{\mu_{Z_{0}}^{2}}{\sigma_{Z_{0}}^{2}}, \quad \Omega_{0}=\frac{\sigma_{Z_{0}}^{2}}{\mu_{Z_{0}}}
\end{aligned}
$$

Then, by following (34), the miss-probability can be derived as

$$
\begin{aligned}
P_{M} & =\frac{1}{2^{L}} \sum_{\boldsymbol{b}_{L} \in \mathcal{B}^{L}} P\left(Z_{u} \leq C_{T} \mid \boldsymbol{b}_{L}, b_{u}=1\right) \\
& =\frac{1}{2^{L}} \sum_{\boldsymbol{b}_{L} \in \mathcal{B}^{L}} \frac{1}{\Gamma\left(m_{1}\right) \Omega_{1}^{m_{1}}} \int_{0}^{C_{T}} y^{m_{1}-1} \exp \left(-\frac{y}{\Omega_{1}}\right) d y
\end{aligned}
$$

Using the result of (3.381.1) in [44], we can simplify the above expression to

$$
P_{M}=\frac{1}{2^{L}} \sum_{\boldsymbol{b}_{L} \in \mathcal{B}^{L}} \frac{\gamma\left(m_{1}, C_{T} / \Omega_{1}\right)}{\Gamma\left(m_{1}\right)}
$$

where $\gamma(\alpha, x)$ is the lower incomplete gamma function [44](8.350.1). Furthermore, when $m_{1}$ is an integer, or when it is approximated by an integer ${ }^{1}$, then, with the aid of [44](8.352.6), (42) can be expressed as

$$
P_{M}=\frac{1}{2^{L}} \sum_{\boldsymbol{b}_{L} \in \mathcal{B}^{L}}\left[1-\exp \left(-\frac{C_{T}}{\Omega_{1}}\right) \sum_{n=0}^{m_{1}-1} \frac{1}{n !}\left(\frac{C_{T}}{m_{1}}\right)^{n}\right]
$$

Similarly, corresponding to (42) and (43), the false-alarm

\footnotetext{
${ }^{1}$ When $m_{1}$ is large, it usually can be approximated by an integer, as the Gamma-distribution in this case is not very sensitive to value of $m_{1}$.
} 
probability can be expressed as

$$
\begin{aligned}
P_{F A} & =1-\frac{1}{2^{L}} \sum_{\boldsymbol{b}_{L} \in \mathcal{B}^{L}} \frac{\gamma\left(m_{0}, C_{T} / \Omega_{0}\right)}{\Gamma\left(m_{0}\right)} \\
& =1-\frac{1}{2^{L}} \sum_{\boldsymbol{b}_{L} \in \mathcal{B}^{L}}\left[1-\exp \left(-\frac{C_{T}}{\Omega_{0}}\right) \sum_{n=0}^{m_{0}-1} \frac{1}{n !}\left(\frac{C_{T}}{m_{0}}\right)^{n}\right]
\end{aligned}
$$

respectively.

Finally, in the general cases, when substituting (42) and (44) into (25), we can obtain the BER expression for the OOKmodulated DMC systems under the Gamma-approximation. By contrast, for the special cases of $m_{1}$ and $m_{0}$ being integers, corresponding BER expression can be obtained by substituting (43) and (45) into (25).

\section{Monte-Carlo Approach}

So far, we have considered some alternative approaches to deal with the second sum in (31). In some cases, such as when communicating at relatively high data rate, the ISI can be very long, which makes exact evaluation of (31) impossible, due to the involvement of the first sum of $2^{L}$ items, as seen in (31). In fact, provided that $L \geq 20$, exact evaluating (31) is highly involved, even without considering the second sum in (31). In practice, however, the ISI length of $L$ may be significantly larger than 20. Therefore, in this and the next subsections, we provide some approaches for reducing the terms to be considered in the context of the first sum in (31), as well as in the BER expressions obtained from the Gaussian- and Gamma-approximation.

Specifically, in this subsection, we consider the Monte-Carlo approach [45] to simplify the computation of the BER expressions obtained under the Poisson, Gaussian-approximation and Gamma-approximation approaches, when $L$ is large. As seen in (31), since $\boldsymbol{b}_{L}$ is a $L$-length binary random vector distributed in $\mathcal{B}^{L}$, we can randomly generate $W$ number of binary sequences for $\boldsymbol{b}_{L}$, which are expressed as $\left\{\boldsymbol{b}_{L}^{(1)}, \ldots, \boldsymbol{b}_{L}^{(W)}\right\}$. Then, corresponding to (31), the BER of the DMC systems employing OOK modulation can be approximately evaluated from the formula

$$
P_{b} \approx \frac{1}{2}+\frac{1}{2 W} \sum_{\left\{\boldsymbol{b}_{L}^{(1)}, \ldots, \boldsymbol{b}_{L}^{(W)}\right\}} \sum_{n=0}^{\left\lceil V_{R} C_{T}\right\rceil}\left(\frac{\mu_{Y_{1}}^{n} e^{-\mu_{Y_{1}}}-\mu_{Y_{0}}^{n} e^{-\mu_{Y_{0}}}}{n !}\right)
$$

Provided that $W$ is sufficiently large, the BER estimated by (46) should be close to that by (31).

Similarly, we can have the approximated BER expressions, when the Gaussian- and Gamma-approximation are respectively employed. As our results in Section V show, the MonteCarlo approach is capable of providing close approximation, provided that $W \geq 10^{5}$.

\section{E. Simplified Poisson, Gaussian-Approximation and Gamma- Approximation Approaches}

From Fig. 1 we can see that the concentration becomes flatter and lower, as the propagation time or/and propagation distance increase. This implies that the ISI imposing on a specific data bit is dominated by the several bits sent in the front of the bit. Therefore, when $L$ is large, and assuming that $u>>1$ and $u>L$, we may re-write the decision variable $Z_{u}$ of (5) as

$$
Z_{u}=\sum_{i=0}^{I} b_{u-i}\left[c_{i}+n_{(u-i) u}\right]+X_{u}, \quad u=0,1, \ldots
$$

where $X_{u}=\sum_{i=I+1}^{L} b_{u-i}\left[c_{i}+n_{(u-i) u}\right]$. Since all $c_{i}$ in $X_{u}$ have small values and $n_{(u-i) u}$ in $X_{u}$ are independent random variables, when $(L-I)$ is sufficiently large, we may approximate $Y_{u}=V_{R} X_{u}$ as a Poisson distributed random variable, with the mean and variance given by

$$
\mu_{y}=E\left[V_{R} X_{u}\right]=V_{R} E_{\left\{b_{u-i}\right\}}\left[\sum_{i=I+1}^{L} b_{u-i} c_{i}\right]=\frac{V_{R}}{2} \sum_{i=I+1}^{L} c_{i}
$$

Therefore, $X_{u}$ has the mean and variance given by

$$
\mu_{x}=\frac{1}{2} \sum_{i=I+1}^{L} c_{i}, \quad \sigma_{x}^{2}=\frac{1}{2 V_{R}} \sum_{i=I+1}^{L} c_{i}
$$

Consequently, when the Poisson approach is employed, the PDFs of $Y_{u}$ conditioned on $b_{u}=1$ and $b_{u}=0$ are given by (23) and (24), respectively, associated with

$$
\begin{aligned}
& \mu_{Y_{1}}=V_{R}\left(c_{0}+\sum_{i=1}^{I} b_{u-i} c_{i}\right)+\mu_{y}, \\
& \mu_{Y_{0}}=V_{R} \sum_{i=1}^{I} b_{u-i} c_{i}+\mu_{y}
\end{aligned}
$$

Finally, following the derivation of (31), the BER of the OOK-modulated DMC systems derived from the simplified Poisson approach can be expressed as (31) with the parameter $L$ replaced by the parameter $I$, and $b_{L} \in \mathcal{B}^{L}$ replaced by $\boldsymbol{b}_{I} \in \mathcal{B}^{I}$ where, by definition, $\boldsymbol{b}_{I}=\left[b_{u-I}, \ldots, b_{u-1}\right]$.

When the simplified Gaussian-approximation is employed, the conditional PDFs of $Z_{u}$ are respectively given in (32) for $b_{u}=1$ and $b_{u}=0$, associated with

$$
\begin{aligned}
& \mu_{Z_{1}}=c_{0}+\sum_{i=1}^{I} b_{u-i} c_{i}+\mu_{x} ; \quad \sigma_{Z_{1}}^{2}=\frac{c_{0}}{V_{R}}+\frac{1}{V_{R}} \sum_{i=1}^{I} b_{u-i} c_{i}+\sigma_{x}^{2} \\
& \mu_{Z_{0}}=\sum_{i=1}^{I} b_{u-i} c_{i}+\mu_{x} ; \quad \sigma_{Z_{0}}^{2}=\frac{1}{V_{R}} \sum_{i=1}^{I} b_{u-i} c_{i}+\sigma_{x}^{2}
\end{aligned}
$$

Correspondingly, the BER of the OOK-modulated DMC systems can be derived by following that in Section III-B, which can be expressed as

$$
P_{b}=\frac{1}{2}+\frac{1}{2^{I+1}} \sum_{\boldsymbol{b}_{I} \in \mathcal{B}^{I}}\left[Q\left(\frac{C_{T}-\mu_{Z_{0}}}{\sigma_{Z_{0}}}\right)-Q\left(\frac{C_{T}-\mu_{Z_{1}}}{\sigma_{Z_{1}}}\right)\right]
$$

Finally, when the simplified Gamma-approximation is employed, following the analysis in Section III-B, we can show 
that the BER of the OOK-modulated DMC systems can be formulated as

$$
P_{b}=\frac{1}{2}+\frac{1}{2^{I+1}} \sum_{\boldsymbol{b}_{I} \in \mathcal{B}^{I}}\left[\frac{\gamma\left(m_{1}, C_{T} / \Omega_{1}\right)}{\Gamma\left(m_{1}\right)}-\frac{\gamma\left(m_{0}, C_{T} / \Omega_{0}\right)}{\Gamma\left(m_{0}\right)}\right]
$$

where $m_{1}, m_{0}$ and $\Omega_{1}, \Omega_{0}$ are given in (40) with $\mu_{Z_{1}}, \mu_{Z_{0}}$ and $\sigma_{Z_{1}}^{2}, \sigma_{Z_{0}}^{2}$ given in (51).

In comparison to the BER formulas in Sections III-A, III-B and III-C, which need to consider $2^{L}$ terms for the first sum at the RHS of the BER formulas, as seen, e.g., in (31), we now only need to compute $2^{I}$ terms. In Section V, we will demonstrate the effect of the value of $I$ on the accuracy of evaluated BER, showing that we can choose $I<<L$, when $L$ is large. Typically, $I \approx 10$ is sufficient for obtaining nearaccurate BER evaluation, provided that the data rate is not too high and, hence, the ISI imposed by any of the other $(L-I)$ data bits is insignificant. Hence, significantly reduced computation is possible, while still achieving sufficiently accurate evaluation.

\section{BER ANALYSIS OF DMC SySTEMS WITH OOK MODULATION AND ISI CANCELLATION}

In this section, we analyze the BER of the OOK-modulated DMC systems with the ISIC. Our analysis starts with the decision variable $Z_{u}$ given by (12), and assumes that $\lambda_{u}=\bar{\lambda}$ is a constant. Furthermore, as in Section III, we assume that the maximum ISI length is $L$ bits, and that $u>L$ is considered. Then, $Z_{u}$ can be written as

$$
\begin{aligned}
Z_{u}= & \underbrace{\sum_{i=0}^{L} b_{u-i}\left[c\left(i T+\hat{t}_{d}\right)+n_{u-i}\left(u T+\hat{t}_{d}\right)\right]}_{Z_{u d}} \\
& -\underbrace{\bar{\lambda} \times \sum_{i=1}^{L} b_{u-i}\left[c(i T)+n_{u-i}(u T)\right]}_{Z_{u 0}}
\end{aligned}
$$

Let $c_{i}=c\left(i T+\hat{t}_{d}\right), n_{(u-i) u}=n_{u-i}\left(u T+\hat{t}_{d}\right)$ and $c_{i}^{\prime}=$ $c(i T), n_{(u-i) u}^{\prime}=n_{u-i}(u T)$. Then, the above equation can be re-written as

$$
\begin{aligned}
Z_{u}= & \underbrace{\sum_{i=0}^{L} b_{u-i} c_{i}+\sum_{i=0}^{L} b_{u-i} n_{(u-i) u}}_{Z_{u d}} \\
& -\underbrace{-\bar{\lambda} \times\left[\sum_{i=1}^{L} b_{u-i} c_{i}^{\prime}+\sum_{i=1}^{L} b_{u-i} n_{(u-i) u}^{\prime}\right]}_{Z_{u 0}}
\end{aligned}
$$

In order for the analysis to be manageable, we assume that $t_{d}>\rho^{2} / D$, so that the two noise samples obtained at $t=$ $u T+\hat{t}_{d}$ and $t=u T$ are independent [35].

In this section, we derive the BER formulas, when the Poisson approach, simplified Poisson approach, Gaussianapproximation, and simplified Gaussian-approximation are considered. Unfortunately, the PDF for the difference of two Gamma variates distributed with different shaping parameters is unknown in public references, as claimed in [46], the Gamma-approximation approaches will hence not be considered in this section.

\section{A. Poisson and Simplified Poisson Approaches}

Following our analysis in Section III-A, it can be shown that, conditioned on $\boldsymbol{b}_{L}$ and $b_{u}=1, Y_{u d}=V_{R} Z_{u d}$ obeys the Poisson PMF of

$$
f_{Y_{u d}}\left(n \mid \boldsymbol{b}_{L}, b_{u}=1\right)=\frac{\mu_{Y_{1}}^{n} e^{-\mu_{Y_{1}}}}{n !}, n=0,1,2, \ldots
$$

where $\mu_{Y_{1}}=V_{R}\left(c_{0}+\sum_{i=1}^{L} b_{u-i} c_{i}\right)$. By contrast, conditioned on $\boldsymbol{b}_{L}$ and $b_{u}=0, Y_{u d}$ obeys the Poisson PMF of

$$
f_{Y_{u d}}\left(n \mid \boldsymbol{b}_{L}, b_{u}=0\right)=\frac{\mu_{Y_{0}}^{n} e^{-\mu_{Y_{0}}}}{n !}, n=0,1,2, \ldots
$$

where $\mu_{Y_{0}}=V_{R} \sum_{i=1}^{L} b_{u-i} c_{i}$. As seen in (55), $Z_{u 0}$ is only dependent on $\boldsymbol{b}_{L}$ but not on $b_{u}$. Hence, the conditional PMF of $Y_{u 0}=V_{R} Z_{u 0}$ can be expressed as

$$
f_{Y_{u 0}}\left(n \mid \boldsymbol{b}_{L}\right)=\frac{\mu_{Y}^{n} e^{-\mu_{Y}}}{n !}, n=0,1,2, \ldots
$$

where $\mu_{Y}=V_{R} \bar{\lambda} \sum_{i=1}^{L} b_{u-i} c_{i}^{\prime}$.

Since the noise samples in $Z_{u d}$ and $Z_{u 0}$ are independent, the conditional PMFs of $f_{Y_{u d}}\left(n \mid \boldsymbol{b}_{L}, b_{u}=1\right.$ ) (or $f_{Y_{u d}}\left(n \mid \boldsymbol{b}_{L}, b_{u}=\right.$ $0)$ ) and $f_{Y_{u 0}}\left(n \mid \boldsymbol{b}_{L}\right)$ are independent. According to the properties of the Poisson distribution, the difference of two independent Poisson distributions obeys the Skellam distribution [47]. Hence, the PMF of $Y_{u}=V_{R} Z_{u}$ conditioned on $\left(\boldsymbol{b}_{L}, b_{u}=1\right)$ and $\left(\boldsymbol{b}_{L}, b_{u}=0\right)$ can be expressed as

$$
\begin{aligned}
& f_{Y_{u}}\left(k \mid b_{L}, b_{u}=1\right)=\exp \left(-\left[\mu_{Y_{1}}+\mu_{Y}\right]\right)\left(\frac{\mu_{Y_{1}}}{\mu_{Y}}\right)^{k / 2} \\
& \quad \times I_{k}\left(2 \sqrt{\mu_{Y_{1}} \mu_{Y}}\right), \quad-\infty<k<\infty \\
& f_{Y_{u}}\left(k \mid b_{L}, b_{u}=0\right)=\exp \left(-\left[\mu_{Y_{0}}+\mu_{Y}\right]\right)\left(\frac{\mu_{Y_{0}}}{\mu_{Y}}\right)^{k / 2} \\
& \quad \times I_{k}\left(2 \sqrt{\mu_{Y_{0}} \mu_{Y}}\right), \quad-\infty<k<\infty
\end{aligned}
$$

respectively. In the above equations, $I_{k}(z)$ is the modified Bessel function [47].

Having obtained the PMFs of $Y_{u}$, as shown in (59), then, given a decision threshold $C_{T}$ in the sense of concentration, the miss-probability can be derived as

$$
\begin{aligned}
P_{M}= & \frac{1}{2^{L}} \sum_{\boldsymbol{b}_{L} \in \mathcal{B}^{L}} P\left(Y_{u} \leq V_{R} C_{T} \mid \boldsymbol{b}_{L}, b_{u}=1\right) \\
= & \frac{1}{2^{L}} \sum_{\boldsymbol{b}_{L} \in \mathcal{B}^{L}} \exp \left(-\left[\mu_{Y_{1}}+\mu_{Y}\right]\right) \\
& \times \sum_{k=-\infty}^{\left\lceil V_{R} C_{T}\right\rceil}\left(\frac{\mu_{Y_{1}}}{\mu_{Y}}\right)^{k / 2} I_{k}\left(2 \sqrt{\mu_{Y_{1}} \mu_{Y}}\right)
\end{aligned}
$$


Similarly, for a given decision threshold $C_{T}$, the false-alarm probability can be derived as

$$
\begin{aligned}
P_{F A}= & -\frac{1}{2^{L}} \sum_{\boldsymbol{b}_{L} \in \mathcal{B}^{L}} P\left(Y_{u} \leq V_{R} C_{T} \mid \boldsymbol{b}_{L}, b_{u}=0\right) \\
= & 1-\frac{1}{2^{L}} \sum_{\boldsymbol{b}_{L} \in \mathcal{B}^{L}} \exp \left(-\left[\mu_{Y_{0}}+\mu_{Y}\right]\right) \sum_{k=-\infty}^{\left\lceil V_{R} C_{T}\right\rceil}\left(\frac{\mu_{Y_{0}}}{\mu_{Y}}\right)^{k / 2} \\
& \times I_{k}\left(2 \sqrt{\mu_{Y_{0}} \mu_{Y}}\right)
\end{aligned}
$$

Finally, the BER of the OOK-modulated DMC with ISIC can be expressed as

$$
\begin{aligned}
P_{b}= & \frac{1}{2}\left(P_{M}+P_{F A}\right) \\
= & \frac{1}{2}+\frac{1}{2^{L+1}} \sum_{\boldsymbol{b}_{L} \in \mathcal{B}^{L}} \exp \left(-\mu_{Y}\right)\left[\exp \left(-\mu_{Y_{1}}\right)\right. \\
& \times \sum_{k=-\infty}^{\left\lceil V_{R} C_{T}\right\rceil}\left(\frac{\mu_{Y_{1}}}{\mu_{Y}}\right)^{k / 2} I_{k}\left(2 \sqrt{\mu_{Y_{1}} \mu_{Y}}\right) \\
& \left.-\exp \left(-\mu_{Y_{0}}\right) \sum_{k=-\infty}^{\left\lceil V_{R} C_{T}\right\rceil}\left(\frac{\mu_{Y_{0}}}{\mu_{Y}}\right)^{k / 2} I_{k}\left(2 \sqrt{\mu_{Y_{0}} \mu_{Y}}\right)\right]
\end{aligned}
$$

In the cases that $L$ is large, the BER $P_{b}$ can be evaluated by the Monte-Carlo approach addressed in Section III-D or the simplified Poisson approach analyzed in Section III-E. Specifically, when the simplified Poisson approach is desired, the BER has the expression of (62) with the following modifications. First, $\boldsymbol{b}_{L} \in \mathcal{B}^{L}$ is replaced by $\boldsymbol{b}_{I} \in \mathcal{B}^{I}$, where $\boldsymbol{b}_{I}=\left[b_{u-I}, \ldots, b_{u-1}\right]$, when assuming that there are $I$ bits dominating the ISI on the $u$ th bit. Second, $\mu_{Y_{1}}, \mu_{Y_{0}}$ and $\mu_{Y}$ are respectively replaced by the quantities of

$$
\begin{aligned}
\mu_{Y_{1}} & =V_{R}\left(c_{0}+\sum_{i=1}^{I} b_{u-i} c_{i}+\frac{1}{2} \sum_{i=I+1}^{L} c_{i}\right), \\
\mu_{Y_{0}} & =V_{R}\left(\sum_{i=1}^{I} b_{u-i} c_{i}+\frac{1}{2} \sum_{i=I+1}^{L} c_{i}\right), \\
\mu_{Y} & =V_{R} \bar{\lambda}\left(\sum_{i=1}^{I} b_{u-i} c_{i}^{\prime}+\frac{1}{2} \sum_{i=I+1}^{L} c_{i}^{\prime}\right)
\end{aligned}
$$

\section{B. Gaussian-Approximation and Simplified Gaussian- Approximation}

According to [48], when both $\left(\mu_{Y_{1}}+\mu_{Y}\right)$ and $\left(\mu_{Y_{0}}+\mu_{Y}\right)$ are relatively large, the Skellam PMFs in (59) can be approximated by the corresponding Gaussian PDFs. When we directly deal with concentration and have the decision variable given by (55), the conditional Gaussian PDFs can be respectively expressed as

$$
\begin{aligned}
& f_{Z_{u}}\left(y \mid \boldsymbol{b}_{L}, b_{u}=1\right)=\frac{1}{\sqrt{2 \pi} \sigma_{Z_{1}}} \exp \left(-\frac{\left[y-\mu_{Z_{1}}\right]^{2}}{2 \sigma_{Z_{1}}^{2}}\right) \\
& f_{Z_{u}}\left(y \mid \boldsymbol{b}_{L}, b_{u}=0\right)=\frac{1}{\sqrt{2 \pi} \sigma_{Z_{0}}} \exp \left(-\frac{\left[y-\mu_{Z_{0}}\right]^{2}}{2 \sigma_{Z_{0}}^{2}}\right)
\end{aligned}
$$

where $-\infty<y<\infty$, by definition,

$$
\begin{aligned}
\mu_{Z_{1}} & =\frac{1}{V_{R}}\left(\mu_{Y_{1}}-\mu_{Y}\right)=c_{0}+\sum_{i=1}^{L} b_{u-i}\left(c_{i}-\bar{\lambda} c_{i}^{\prime}\right), \\
\sigma_{Z_{1}}^{2}=\frac{1}{V_{R}^{2}}\left(\mu_{Y_{1}}+\mu_{Y}\right) & =\frac{1}{V_{R}}\left[c_{0}+\sum_{i=1}^{L} b_{u-i}\left(c_{i}+\bar{\lambda} c_{i}^{\prime}\right)\right], \\
\mu_{Z_{0}}=\frac{1}{V_{R}}\left(\mu_{Y_{0}}-\mu_{Y}\right) & =\sum_{i=1}^{L} b_{u-i}\left(c_{i}-\bar{\lambda} c_{i}^{\prime}\right), \\
\sigma_{Z_{0}}^{2}=\frac{1}{V_{R}^{2}}\left(\mu_{Y_{0}}+\mu_{Y}\right) & =\frac{1}{V_{R}}\left[\sum_{i=1}^{L} b_{u-i}\left(c_{i}+\bar{\lambda} c_{i}^{\prime}\right)\right]
\end{aligned}
$$

As seen from the above equations, when the ISI is ideally cancelled, we have $V_{R} \mu_{Z_{1}}=V_{R} c_{0}$, which represents the average number of molecules in the detection space, and $V_{R} \mu_{Z_{0}}=0$. Hence, for the Skellam distribution to be approximated by the Gaussian distribution, it requires that $V_{R} \mu_{Z_{1}}$ is sufficiently large.

Explicitly, the PDFs in (64) have the same forms as those in (32) in Section III-B. Hence, when given the decision threshold of $C_{T}$, the BER of the OOK-modulated DMC systems with ISIC has the same expression as that in Section III-B, with $\mu_{Z_{0}}, \sigma_{Z_{0}}$ and $\mu_{Z_{1}}, \sigma_{Z_{1}}$ replaced by the corresponding terms given in (65).

Furthermore, when the simplified Gaussian-approximation is employed, the BER of the OOK-modulated DMC systems with ISIC can be expressed as (52), with $\mu_{Z_{0}}, \sigma_{Z_{0}}$ and $\mu_{Z_{1}}, \sigma_{Z_{1}}$ provided by the formulas of

$$
\begin{aligned}
& \mu_{Z_{1}}=c_{0}+\sum_{i=1}^{I} b_{u-i}\left(c_{i}-\bar{\lambda} c_{i}^{\prime}\right)+\frac{1}{2} \sum_{i=I+1}^{L}\left(c_{i}-\bar{\lambda} c_{i}^{\prime}\right), \\
& \sigma_{Z_{1}}^{2}=\frac{1}{V_{R}}\left[c_{0}+\sum_{i=1}^{I} b_{u-i}\left(c_{i}+\bar{\lambda} c_{i}^{\prime}\right)+\frac{1}{2} \sum_{i=I+1}^{L}\left(c_{i}+\bar{\lambda} c_{i}^{\prime}\right)\right], \\
& \mu_{Z_{0}}=\sum_{i=1}^{I} b_{u-i}\left(c_{i}-\bar{\lambda} c_{i}^{\prime}\right)+\frac{1}{2} \sum_{i=I+1}^{L}\left(c_{i}-\bar{\lambda} c_{i}^{\prime}\right), \\
& \sigma_{Z_{0}}^{2}=\frac{1}{V_{R}}\left[\sum_{i=1}^{I} b_{u-i}\left(c_{i}+\bar{\lambda} c_{i}^{\prime}\right)+\frac{1}{2} \sum_{i=I+1}^{L}\left(c_{i}+\bar{\lambda} c_{i}^{\prime}\right)\right]
\end{aligned}
$$

As seen at the RHS of the above equations, these quantities are dependent on $\boldsymbol{b}_{I}$ containing only $I$ data bits.

\section{Performance Results}

In this section, we demonstrate the BER performance of the OOK-modulated DMC systems with or without ISIC, and compare the results obtained by the various analytical approaches considered in Sections III and IV. Furthermore, some results obtained from both the particle-based simulations and the Monte-Carlo simulations [49-51] are provided to validate the analytical results. In order to demonstrate the results, we define the SNR by following the convention in the traditional wireless communications [37] as the ratio between the power received from a single impulse of molecules and the noise power, which is expressed as

$$
\gamma_{b}=\frac{c_{0}^{2}}{E\left[\sigma_{0}^{2}\right]}=\frac{c_{0}^{2}}{c_{0} / V_{R}}=V_{R} c_{0}
$$




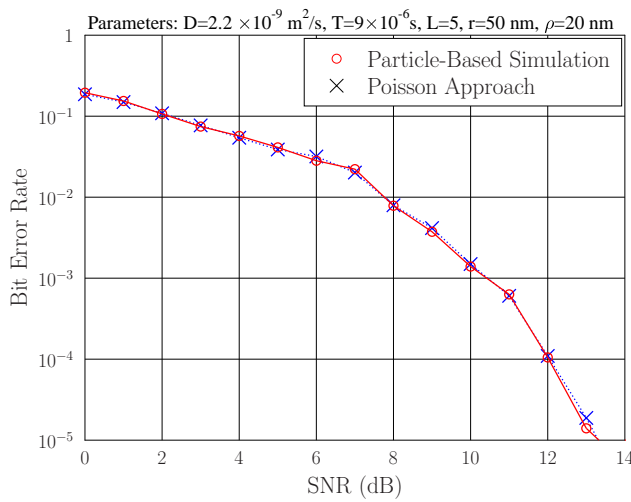

Fig. 3. Comparison of the BER versus SNR performance of the OOKmodulated DMC systems obtained by the particle-based simulations anc Poisson approach.

From this definition and the analysis in Section II, we can know that, given the diffusion coefficient $D$ and the transmission distance $r$, the SNR is only dependent on the number of molecules $Q$ emitted by one pulse and the volume $V_{R}$ of the detection space. In our studies, we set $D=2.2 \times 10^{-9} \mathrm{~m}^{2} / \mathrm{s}$ (squared meter/seconds). For Fig. 3 considering the particlebased simulations, we assume relatively short communications distance and set the radius of the spherical detection space to $\rho=20 \mathrm{~nm}$ (nano meter), as the result that particlebased simulation is difficult to cope with a big number of molecules. By contrast, for all the other figures, we consider relatively long communications distance, and set the radius of the spherical detection space to $\rho=1 \mathrm{~nm}$. Furthermore, in our studies, the ISI length $L$ is estimated as

$$
L \triangleq \arg _{l}\left\{c_{l} / c_{0} \leq 0.1 \%\right\}
$$

meaning that the ISI from the received pulses having their peak concentration at least 1000 times lower than that of the current pulse is ignored.

Additionally, we note that, for convenience, the parameters used for generating the results are specified with the figures. Furthermore, unless notified, such as in Figs. 9 and 10, the results are obtained, when the optimum detection thresholds are applied.

First, in Figs. 3 and 4, we compare the BER versus SNR performance obtained from the analytical and simulation approaches. Specifically, in Fig.3, the analytical results were obtained from the Poisson approach of Section III-A, which are compared with the corresponding results obtained from the particle-based simulations. By contrast, in Fig. 4, we demonstrate the BER versus SNR performance of the OOKmodulated DMC systems, when the systems are operated with different data rates reflected by the different bit-durations of $T$. Correspondingly, the length of ISI in different cases is different, as shown in the figure. In the figure, the analytical results were obtained from the Poisson approach of Section III-A for the cases of $L=10$ and $L=20$, while obtained from the Poisson Monte-Carlo approach of Section III-D for the other cases of $L=30-80$. Note that here the Poisson Monte-Carlo approach was used because, when $L \geq 30$, it is very time-

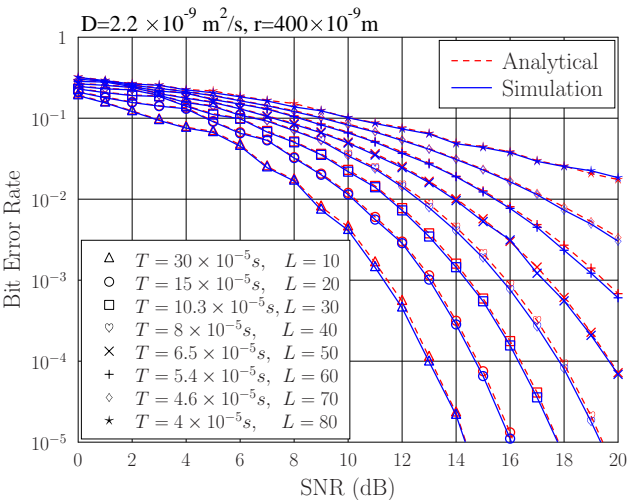

Fig. 4. Comparison of the BER versus SNR performance of the OOKmodulated DMC systems obtained by the Monte-Carlo simulations and the Poisson or Poisson (Monte-Carlo) approach.

consuming to compute the results by the Poisson approach of Section III-A. The simulation results shown in Fig. 4 were obtained from the Monte-Carlo simulations. From the results of Figs. 3 and 4 we may have the following observations. First, the analytical results agree closely with the simulation results obtained from either the particle-based or the Monte-Carlo simulations. Therefore, the Poisson modelling considered in this paper is accurate and that the Poisson and Poisson MonteCarlo approaches are valid. Second, as shown in Fig. 4, when the symbol duration decreases, i.e., the data rate increases, the BER performance of the DMC systems degrades due to the increased ISI.

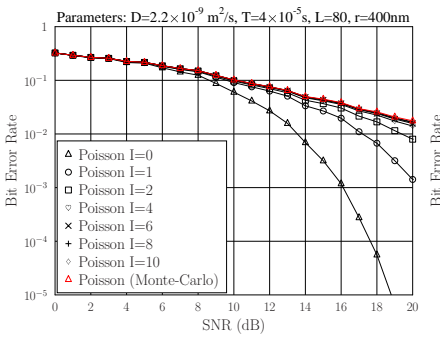

(a) OOK Modulation

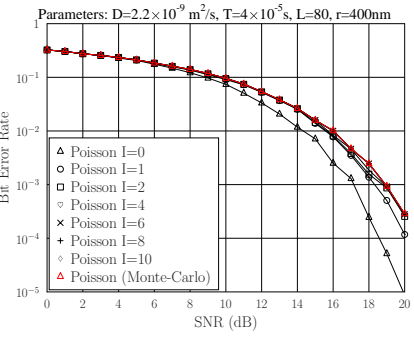

(b) OOK modulation with ISIC
Fig. 5. Impact of parameter $I$ on the accuracy of the BER versus SNR performance of the DMC systems estimated by the simplified Poisson approach.

In Fig. 5, we show the impact of the parameter $I$ used in the simplified Poisson (Gaussian, and Gamma) approach on the accuracy of the estimated BER performance of the DMC systems, when the OOK modulation without ISIC (Fig. 5(a)) and that with ISIC (Fig. 5(b)) are employed. Note that, here we consider only the simplified Poisson approach, as our results showed that the simplified Gaussian and Gamma approaches appear the same behavior as the simplified Poisson approach. From the results of Fig. 5, we observe that, for both the cases of without/with ISIC, the BER performance evaluated by the simplified approach converges to that obtained by the MonteCarlo approach, provided that $I \geq 6$ in Fig. 5(a) and $I \geq 2$ in Fig. 5(b). This observation implies that, when $L$ is relatively 


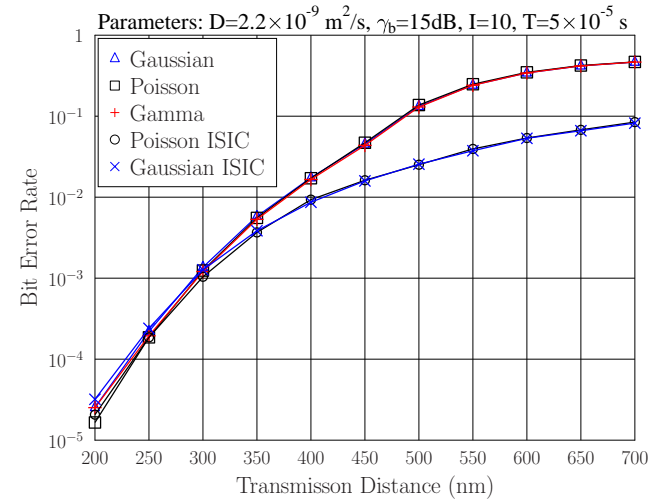

Fig. 6. Comparison of different approaches for the BER versus transmission distance of the DMC systems with OOK modulation and without/with ISIC.

large, e.g., $L>20$, those less dominate ISI can be effectively approximated by a random variable without depending on the specific bit sequence. Hence, the simplified approaches can be efficiently employed for evaluating the error performance of the DMC systems. We should note that in Figs. 5(a) and 5(b), the Monte-Carlo Poisson approach was used instead of the exact Poisson approach, because it is impossible to evaluate the BER by the exact Poisson approach in the case of $L=80$, which corresponds to $2^{80}$ combinations of bit sequences.

When comparing the results (red-colored lines) in Figs. 5(a) and 5(b), we can observe that the ISIC is capable of providing some performance gain, as shown also by the forthcoming results.

Fig. 6 shows the BER performance of the DMC systems without ISIC (top three curves) and that with ISIC (bottom two curves) against the transmission distance, where the results were evaluated by the different approaches as specified in the figure. Explicitly, both the Gaussian- and Gamma-approximation are accurate, which give nearly the same performance results as the exact Poisson approach. As shown in Fig. 6, the performance gain provided by the ISIC becomes higher, as the transmission distance increases. The reason behind is that, given the symbol duration $T$, the ISI increases as the result that the increase of transmission distance results in the expansion of the received pulse, as seen in Fig. 1. There is no performance gain available by applying the ISIC, when the transmission distance is below $300 \mathrm{~nm}$. In fact, when the transmission distance is short, e.g., 200nm, the ISIC generates negative performance gain. This is because ISIC amplifies noise, as seen in (9), due to the randomness of the noise samples.

In Fig. 7, we demonstrate the BER versus SNR performance of the DMC systems without/with ISIC, estimated by the different approaches. Notice in the figures that the sets of curves are distinguished by the transmission distances $r$ and their corresponding ISI length. When comparing the two figures, we can see that the employment of ISIC results in more reliable detection. More performance gain is available, as the transmission distance increases, which follows the observation in Fig. 6. In both figures, the BER performance degrades

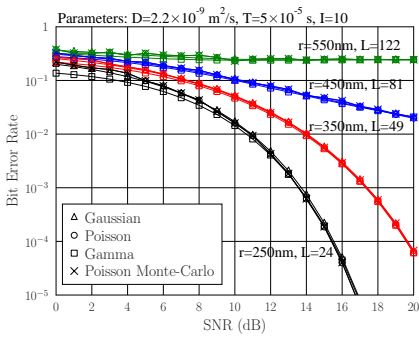

(a) OOK Modulation

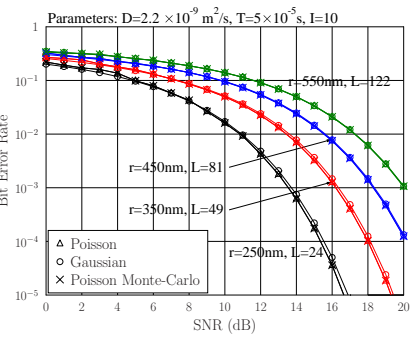

(b) OOK modulation with ISIC

Fig. 7. Comparison of different approaches for the BER versus SNR performance of the DMC systems without/with ISIC.

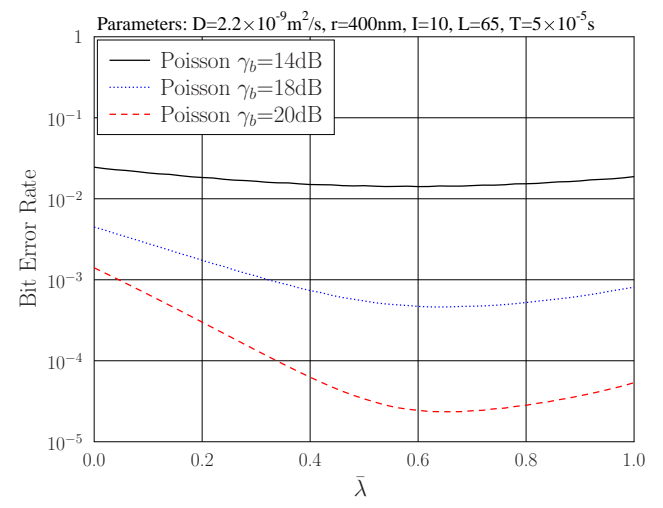

Fig. 8. BER versus ISIC scaling factor $\bar{\lambda}$ of the OOK-modulated DMC systems with ISIC.

with the increase of transmission distance. Furthermore, all the optional approaches provide near-exact results. However, in the case of without ISIC, when the transmission distance is short, e.g., $250 \mathrm{~nm}$, the BER evaluated by the Gammaapproximation yields marginal difference from the exact one. This is more declared, when the SNR becomes lower. In the case of Fig. 7(b) with ISIC, when the transmission distance is relatively short, e.g., $250 \mathrm{~nm}$, the result evaluated by the Gaussian-approximation has a slight difference from the exact one.

In Fig. 8, we show the effect of the ISIC scaling factor $\bar{\lambda}$ on the BER performance of the DMC systems with ISIC. Explicitly, given the other system parameters, there is an optimum value for $\bar{\lambda}$, which results in the lowest BER. As seen in Fig. 8, the optimum $\bar{\lambda}$ value is depended on the SNR and is around 0.6 for the cases considered. From these results we are inferred that a good $\bar{\lambda}$ value is around 0.6 for the SNR practically interest, which results in the BER between $10^{-5}$ and $10^{-2}$. Furthermore, the achievable BER performance is not very sensitive to the value of $\bar{\lambda}$, and nearly the same BER performance can be attained, provided that $\bar{\lambda}$ changes within a limited range around the optimum value.

Fig. 9 depicts the BER of the DMC systems versus the detection threshold, when ISIC is not employed (Fig. 9(a)) or employed (Fig. 9(b)). As shown in the figures, three symbol durations are considered, which are $T=30 \mu \mathrm{s}, 50 \mu \mathrm{s}$ and $70 \mu \mathrm{s}$, which for a given transmission distance of $250 \mathrm{~nm}$ yields ISI length of $L=42,24$ and 17, respectively. Furthermore, in the 


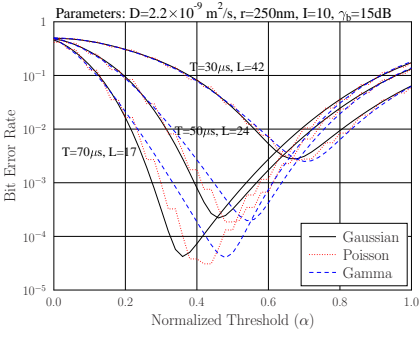

(a) OOK Modulation

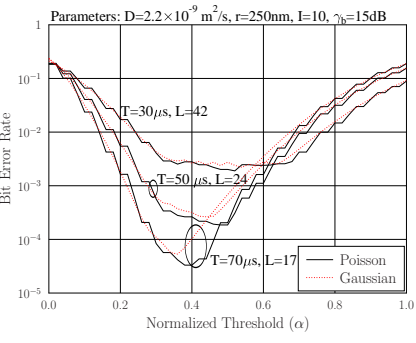

(b) OOK modulation with ISIC
Fig. 9. Comparison of different approaches for the BER versus detection threshold performance of the DMC systems without/with ISIC.

two figures, we compare the BER evaluated by the different approaches, as marked in the figures. From the results, we may have the following observations. First, for given system parameters, there is an optimum threshold, which yields the lowest BER. Second, for the OOK-modulated DMC systems without ISIC, the BER performance is highly sensitive to the threshold. A threshold slightly different from the optimum one might result in a significant increase of BER. Third, when the OOK-modulated DMC systems employ the proposed ISIC, the BER performance becomes less sensitive to the detection threshold. Forth, for the DMC systems without ISIC (Fig. 9(a)), when the optimum thresholds are invoked, both the Gaussian- and Gamma-approximation provide near-accurate approximation to the Poisson approach. By contrast, for the DMC systems with ISIC (Fig. 9(b)), even when operated at the optimum thresholds, the Gaussian-approximation yields a BER higher than that given by the Poisson approach, which is clearly seen in the cases of $T=50 \mu \mathrm{s}$ and $70 \mu \mathrm{s}$. The reason is that, in this case, the ISI is relatively short, resulting in that the condition for applying the Gaussian approximation is not well satisfied. Additionally, as seen in both the figures, the 'optimum threshold' given by the Gaussian or Gamma approach is actually optimum. There is an explicit difference from the optimum threshold given by the Poisson approach.

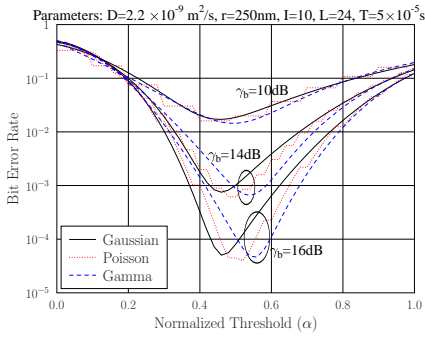

(a) OOK Modulation

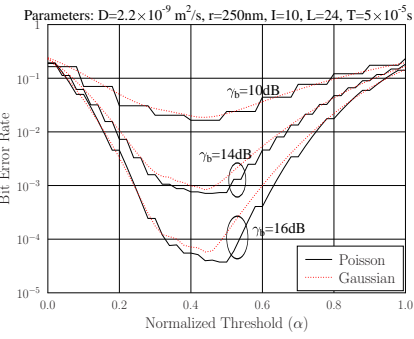

(b) OOK modulation with ISIC
Fig. 10. Comparison of different approaches for the BER versus detection threshold performance of the DMC systems without/with ISIC.

Finally, in Fig. 10, we depict the BER versus detection threshold performance of the DMC systems with respect to $\gamma_{b}=10 \mathrm{~dB}, 14 \mathrm{~dB}$ and $16 \mathrm{~dB}$, when without ISIC (Fig. 10(a)) or with ISIC (Fig. 10(b)). Explicitly, we have similar observations as those from Fig. 9(a) and Fig. 9(b). It is worthy of noting again that the BER performance of the OOK-modulated DMC systems without ISIC is very sensitive to the detection thresh- old applied, and employing ISIC mitigates this sensitivity.

\section{CONCLUSIONS}

We have proposed a range of approaches for analyzing and computing the BER of the DMC systems without or with ISIC, based on which exact and approximate BER expressions have respectively been derived. Our studies and comparison show that both the Gaussian- and Gamma-approximation are capable of providing near-accurate BER estimation, provided that the respective optimum thresholds are applied in detection. However, the optimum detection threshold estimated by the Gaussian- or Gamma-approximation is explicitly different from that given by the Poisson modeling, and hence it is practically not optimum. The BER performance of the OOKmodulated DMC systems without ISIC is very sensitive to the change of detection threshold, whereas, the ISIC is able to mitigate the sensitivity to the variation of detection threshold. Furthermore, the employment of ISIC is able to improve the BER performance of the OOK-modulated DMC systems, especially in the scenarios where transmission distance is relatively long or/and transmission rate is high, both resulting in long ISI. However, ISIC amplifies the counting noise, which may result in performance degradation in the case of short ISI. We have shown that, when ISI is long, the Monte-Carlo approach or the different types of simplified approaches may be employed for evaluating the BER of the DMC systems without or with ISIC. The studies and performance results show that all these approaches are effective approaches, and are capable of providing near-accurate BER estimation.

\section{ACKNOWLEDGEMENT}

The authors would like to thank the anonymous reviewers for their valuable comments and suggestions, which led to a significant improvement of the paper.

\section{REFERENCES}

[1] L. Shi and L. L. Yang, "Diffusion-based molecular communications: Inter-symbol interference cancellation and system performance," in 2016 IEEE/CIC International Conference on Communications in China (ICCC), July 2016, pp. 1-6.

[2] I. F. Akyildiz, F. Brunetti, and C. Blázquez, "Nanonetworks: A new communication paradigm," Comput. Netw., vol. 52, no. 12, pp. 22602279, Aug. 2008.

[3] L. Parcerisa Giné and I. F. Akyildiz, "Molecular communication options for long range nanonetworks," Comput. Netw., vol. 53, no. 16, pp. 2753 2766, Nov. 2009.

[4] T. Nakano, M. J. Moore, F. Wei, A. V. Vasilakos, and J. Shuai, "Molecular communication and networking: Opportunities and challenges," IEEE Transactions on NanoBioscience, vol. 11, no. 2, pp. 135-148, June 2012.

[5] T. Nakano, T. Suda, Y. Okaie, M. J. Moore, and A. V. Vasilakos, "Molecular communication among biological nanomachines: A layered architecture and research issues," IEEE Transactions on NanoBioscience, vol. 13, no. 3, pp. 169-197, Sept 2014.

[6] P. C. Yeh, K. C. Chen, Y. C. Lee, L. S. Meng, P. J. Shih, P. Y. Ko, W. A. Lin, and C. H. Lee, "A new frontier of wireless communication theory: Diffusion-based molecular communications," IEEE Wireless Communications, vol. 19, no. 5, pp. 28-35, Oct 2012.

[7] I. F. Akyildiz, F. Fekri, R. Sivakumar, C. R. Forest, and B. K. Hammer, "Monaco: fundamentals of molecular nano-communication networks," IEEE Wireless Communications, vol. 19, no. 5, pp. 12-18, Oct 2012.

[8] N. Farsad, H. B. Yilmaz, A. Eckford, C. B. Chae, and W. Guo, "A comprehensive survey of recent advancements in molecular communication," IEEE Communications Surveys Tutorials, vol. 18, no. 3, pp. 1887-1919, thirdquarter 2016. 
[9] I. Llatser, A. Cabellos-Aparicio, and E. Alarcon, "Networking challenges and principles in diffusion-based molecular communication," IEEE Wireless Communications, vol. 19, no. 5, pp. 36-41, Oct 2012.

[10] B. Atakan and O. B. Akan, "On channel capacity and error compensation in molecular communication," Transactions on Computational Systems Biology X, vol. 10, pp. 59-80, 2008.

[11] _ _ "Deterministic capacity of information flow in molecular nanonetworks," Nano Communication Networks, vol. 1, no. 1, pp. 31 - 42, 2010

[12] M. U. Mahfuz, D. Makrakis, and H. Mouftah, "Spatiotemporal distribution and modulation schemes for concentration-encoded medium-to-long range molecular communication," in 2010 25th Biennial Symposium on Communications, May 2010, pp. 100-105.

[13] I. Llatser, A. Cabellos-Aparicio, M. Pierobon, and E. Alarcon, "Detection techniques for diffusion-based molecular communication," IEEE Journal on Selected Areas in Communications, vol. 31, no. 12, pp. 726734, December 2013.

[14] N. R. Kim and C. B. Chae, "Novel modulation techniques using isomers as messenger molecules for nano communication networks via diffusion," IEEE Journal on Selected Areas in Communications, vol. 31 no. 12, pp. 847-856, December 2013.

[15] M. S. Kuran, H. B. Yilmaz, T. Tugcu, and I. F. Akyildiz, "Modulation techniques for communication via diffusion in nanonetworks," in Communications (ICC), 2011 IEEE International Conference on, June 2011, pp. 1-5.

[16] M. U. Mahfuz, D. Makrakis, and H. T. Mouftah, "Concentrationencoded subdiffusive molecular communication: Theory, channel characteristics, and optimum signal detection," IEEE Transactions on NanoBioscience, vol. 15, no. 6, pp. 533-548, Sept 2016.

[17] _ " "A comprehensive study of sampling-based optimum signal detection in concentration-encoded molecular communication," IEEE Transactions on NanoBioscience, vol. 13, no. 3, pp. 208-222, Sept 2014

[18] M. U. Mahfuz, "Achievable strength-based signal detection in quantityconstrained PAM OOK concentration-encoded molecular communication," IEEE Transactions on NanoBioscience, vol. 15, no. 7, pp. 619626, Oct 2016

[19] S. Kadloor, R. S. Adve, and A. W. Eckford, "Molecular communication using Brownian motion with drift," IEEE Transactions on NanoBioscience, vol. 11, no. 2, pp. 89-99, June 2012.

[20] A. W. Eckford, "Achievable information rates for molecular communication with distinct molecules," in Bio-Inspired Models of Network, Information and Computing Systems, 2007. Bionetics 2007. 2nd, Dec 2007, pp. 313-315.

[21] H. Arjmandi, A. Gohari, M. N. Kenari, and F. Bateni, "Diffusionbased nanonetworking: A new modulation technique and performance analysis," IEEE Communications Letters, vol. 17, no. 4, pp. 645-648, April 2013.

[22] M. H. Kabir, S. M. R. Islam, and K. S. Kwak, "D-MoSK modulation in molecular communications," IEEE Transactions on NanoBioscience, vol. 14, no. 6, pp. 680-683, Sept 2015.

[23] Y. K. Lin, W. A. Lin, C. H. Lee, and P. C. Yeh, "Asynchronous thresholdbased detection for quantity-type-modulated molecular communication systems," IEEE Transactions on Molecular, Biological and Multi-Scale Communications, vol. 1, no. 1, pp. 37-49, March 2015.

[24] W. Guo, T. Asyhari, N. Farsad, H. B. Yilmaz, B. Li, A. Eckford, and C. B. Chae, "Molecular communications: channel model and physica layer techniques," IEEE Wireless Communications, vol. 23, no. 4, pp. 120-127, August 2016.

[25] D. Kilinc and O. B. Akan, "Receiver design for molecular communication," IEEE Journal on Selected Areas in Communications, vol. 31 no. 12, pp. 705-714, December 2013.

[26] L. S. Meng, P. C. Yeh, K. C. Chen, and I. F. Akyildiz, "On receiver design for diffusion-based molecular communication," IEEE Transaction on Signal Processing, vol. 62, no. 22, pp. 6032-6044, Nov 2014.

[27] A. Noel, K. C. Cheung, and R. Schober, "Optimal receiver design for diffusive molecular communication with flow and additive noise," IEEE Transactions on NanoBioscience, vol. 13, no. 3, pp. 350-362, Sept 2014.

[28] C. T. Chou, "A Markovian approach to the optimal demodulation of diffusion-based molecular communication networks," IEEE Transactions on Communications, vol. 63, no. 10, pp. 3728-3743, Oct 2015.

[29] R. Mosayebi, H. Arjmandi, A. Gohari, M. Nasiri-Kenari, and U. Mitra, "Receivers for diffusion-based molecular communication: Exploiting memory and sampling rate," IEEE Journal on Selected Areas in Communications, vol. 32, no. 12, pp. 2368-2380, Dec 2014.

[30] A. Ahmadzadeh, A. Noel, and R. Schober, "Analysis and design of multi-hop diffusion-based molecular communication networks," IEEE Transactions on Molecular, Biological and Multi-Scale Communications, vol. 1, no. 2, pp. 144-157, June 2015.
[31] M. U. Mahfuz, D. Makrakis, and H. T. Mouftah, "A comprehensive analysis of strength-based optimum signal detection in concentrationencoded molecular communication with spike transmission," IEEE Transactions on NanoBioscience, vol. 14, no. 1, pp. 67-83, Jan 2015.

[32] A. Aijaz and A. H. Aghvami, "Error performance of diffusion-based molecular communication using pulse-based modulation," IEEE Transactions on NanoBioscience, vol. 14, no. 1, pp. 146-151, Jan 2015.

[33] A. Singhal, R. Mallik, and B. Lall, "Performance analysis of amplitude modulation schemes for diffusion-based molecular communication," IEEE Transactions on Wireless Communications, vol. 14, no. 10, pp. 5681-5691, Oct 2015.

[34] S. Galmés and B. Atakan, "Performance analysis of diffusion-based molecular communications with memory," IEEE Transactions on Communications, vol. 64, no. 9, pp. 3786-3793, Sept 2016.

[35] M. Pierobon and I. F. Akyildiz, "Diffusion-based noise analysis for molecular communication in nanonetworks," IEEE Transactions on Signal Processing, vol. 59, no. 6, pp. 2532-2547, June 2011.

[36] A. Papoulis, Probability, Random Variables, and Stochastic Processes, 3rd ed. New York: McGraw-Hill, Inc, 1991.

[37] J. G. Proakis, Digital Communications, 5th ed. McGraw Hill, 2007.

[38] J. Shi, C. Dong, and L.-L. Yang, "Performance comparison of cooperative relay links with different relay processing strategies: Nakagami/Gamma approximation approaches," EURASIP Journal on Wireless Communications and Networking, vol. 2014, no. 1, p. 53, 2014. [Online]. Available: http://dx.doi.org/10.1186/1687-1499-2014-53

[39] M. K. Simon and M.-S. Alouini, Digital Communication over Fading Channels, 2nd ed. New York: John Wiley \& Sons, 2005.

[40] N. Nakagami, "The $m$-distribution, a general formula for intensity distribution of rapid fading," in Statistical Methods in Radio Wave Propagation, W. G. Hoffman, Ed. Oxford, England: Pergamon, 1960.

[41] A. F. Molisch, J. R. Foerster, and M. Pendergrass, "Channel models for ultrawideband personal area networks," IEEE Wireless Communications, vol. 10, no. 6, pp. 14-21, December 2003.

[42] A. F. Molisch, "Ultrawideband propagation channels-theory, measurement, and modeling," IEEE Transactions on Vehicular Technology, vol. 54, no. 5, pp. 1528 - 1545, November 2005.

[43] T. S. Rappaport, R. W. H. Jr., R. C. Daniels, and J. N. Murdock, Millimeter Wave Wireless Communications: Systems and Circuits. USA: Prentice Hall, 2015.

[44] I. Gradshteyn and I. Ryzhik, Table of Integrals, Series, and Products, 7th ed. New York, London: Academic Press, Inc, 2007.

[45] X. Wang and H. V. Poor, Wireless Communication Systems - Advanced Techniques for Signal Reception. Prentice Hall, 2003.

[46] H. Holm and M. S. Alouini, "Sum and difference of two squared correlated Nakagami variates in connection with the McKay distribution," IEEE Transactions on Communications, vol. 52, no. 8, pp. 1367-1376, Aug 2004.

[47] J. G. Skellam, "The frequency distribution of the difference between two poisson variates belonging to different populations," Journal of the Royal Statistical Society, vol. 109, no. 3, pp. 296-296, 1946. [Online]. Available: http://www.jstor.org/stable/2981372

[48] D. Karlis and I. Ntzoufras, "Bayesian analysis of the difference of count data," Statistics in Medicine, vol. 25, no. 11, pp. 1885-1905, June 2006

[49] H. B. Yilmaz, N.-R. Kim, and C.-B. Chae, "Effect of ISI mitigation on modulation techniques in molecular communication via diffusion," in Proceedings of ACM The First Annual International Conference on Nanoscale Computing and Communication, ser. NANOCOM' 14. New York, NY, USA: ACM, 2007, pp. 3:1-3:9. [Online]. Available: http://doi.acm.org/10.1145/2619955.2619959

[50] Y. Deng, A. Noel, M. Elkashlan, A. Nallanathan, and K. C. Cheung, "Modeling and simulation of molecular communication systems with a reversible adsorption receiver," IEEE Transactions on Molecular, Biological and Multi-Scale Communications, vol. 1, no. 4, pp. 347-362, Dec 2015.

[51] Y. Deng, A. Noel, W. Guo, A. Nallanathan, and M. Elkashlan, "Analyzing large-scale multiuser molecular communication via 3-D stochastic geometry," IEEE Transactions on Molecular, Biological and Multi-Scale Communications, vol. 3, no. 2, pp. 118-133, June 2017. 


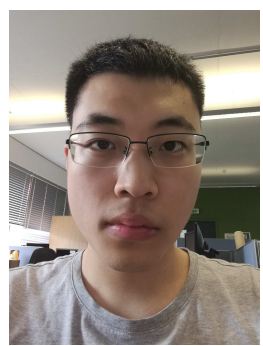

Lu Shi received BEng degree in Electronic Engineering from University of Southampton, Southampton, UK, in 2015. Currently, he is working toward the Ph.D. degree with the University of Southampton. His research is focused on molecular communication systems. His research interests are diffusion-based molecular communication, modulation scheme .

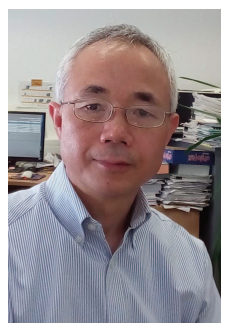

Lie-Liang Yang (M'98, SM'02, F'16) received his BEng degree in communications engineering from Shanghai TieDao University, Shanghai, China in 1988 , and his MEng and PhD degrees in communications and electronics from Northern (Beijing) Jiaotong University, Beijing, China in 1991 and 1997, respectively. From June 1997 to December 1997 he was a visiting scientist of the Institute of Radio Engineering and Electronics, Academy of Sciences of the Czech Republic. Since December 1997, he has been with the University of Southampton, United Kingdom, where he is the professor of wireless communications in the School of Electronics and Computer Science. He has research interest in wireless communications, wireless networks and signal processing for wireless communications, as well as molecular communications and nano-networks. He has published $360+$ research papers in journals and conference proceedings, authored/co-authored three books and also published several book chapters. $\mathrm{He}$ is a fellow of the IET (previously IEE) in the UK, and a distinguished lecturer of the IEEE Vehicular Technology Society. He has served as associate editor to several academic journals, co-organized several special issues, and acted as different roles for conference organization. 